\title{
Ensemble cryo-EM reveals conformational states of the nsp13 helicase in the SARS-CoV-2 helicase replication-transcription complex
}

\author{
James Chen ${ }^{1,6}$, Qi Wang2,6, Brandon Malone ${ }^{1}{ }^{1,6}$, Eliza Llewellyn', Yakov Pechersky ${ }^{2}$, \\ Kashyap Maruthi ${ }^{3}$, Ed T. Eng $\mathbb{D}^{3}$, Jason K. Perry ${ }^{4}{ }^{4}$, Elizabeth A. Campbell', David E. Shaw ${ }^{2,5,7 凶}$ and \\ Seth A. Darst (101,7凶
}

The SARS-CoV-2 nonstructural proteins coordinate genome replication and gene expression. Structural analyses revealed the basis for coupling of the essential nsp13 helicase with the RNA-dependent RNA polymerase (RdRp) where the holo-RdRp and RNA substrate (the replication-transcription complex or RTC) associated with two copies of nsp13 (nsp13 - RTC). One copy of nsp13 interacts with the template-RNA in an opposing polarity to the RdRp and is envisaged to drive the RdRp backward on the RNA template (backtracking), prompting questions as to how the RdRp can efficiently synthesize RNA in the presence of nsp13. Here we use cryogenic-electron microscopy and molecular dynamics simulations to analyze the nsp13 - -RTC, revealing four distinct conformational states of the helicases. The results indicate a mechanism for the nsp13 ${ }_{2}-\mathrm{RTC}$ to turn backtracking on and off, using an allosteric mechanism to switch between RNA synthesis or backtracking in response to stimuli at the RdRp active site.

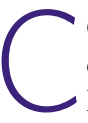
OVID-19, caused by the coronavirus SARS-CoV-2 (refs. ${ }^{1,2}$ ), continues to devastate the world. The viral RNA-dependent RNA polymerase (RdRp, encoded by nonstructural protein 12 or nsp12) functions as a holo-RdRp (comprising nsp $7 / \mathrm{nsp}_{2} / \mathrm{nsp} 12$ ) in a replication-transcription complex (RTC) (holo-RdRp+RNA) to direct RNA synthesis from the viral RNA genome ${ }^{3-5}$. The RdRp is also a target for the clinically approved antiviral remdesivir ${ }^{6-8}$. In addition to the holo-RdRp, the virus encodes several nucleic acid processing enzymes, including a helicase (nsp13), an exonuclease (nsp14), an endonuclease (nsp15) and methyltransferases (nsp14 and nsp 16) ${ }^{9}$. Little is known about how these enzymes coordinate to replicate and transcribe the viral genome.

Nsp13, essential for viral replication ${ }^{10-13}$, is a superfamily $1 \mathrm{~B}$ (SF1B) helicase that can unwind DNA or RNA substrates with a $5^{\prime} \rightarrow 3^{\prime}$ directionality ${ }^{14-16}$. Along with the two canonical RecA ATPase domains of SF1 helicases ${ }^{14,17}$, nsp13 contains three additional domains: an $\mathrm{N}$-terminal zinc-binding domain (unique to nidoviral helicases), a stalk and a $1 \mathrm{~B}$ domain ${ }^{13,18,19}$. Previous studies established that the nsp13 helicase forms a stable complex with the RTC, and single-particle cryogenic-electron microscopy (cryo-EM) structures of an nsp13 - RTC (the RTC with two nsp13 protomers bound) have been determined ${ }^{20-22}$.

In the nsp13 ${ }_{2}-\mathrm{RTC}$ structure, two protomers of nsp13 sit on top of the RTC with each nsp13-zinc-binding domain interacting with one of the two N-terminal helical extensions of nsp8 (refs. ${ }^{20-22}$ ). The two nsp 13 protomers were originally named nsp13.1 and nsp13.2 $\left(\right.$ ref. ${ }^{20}$ ), but we will use the nomenclature proposed by Hillen ${ }^{23}$, nsp $13_{\mathrm{T}}$ (binds on the 'Thumb' side of the RdRp) and nsp13 ${ }_{\mathrm{F}}$ (binds on the 'Fingers' side of the RdRp) (Extended Data Fig. 1). This overall architecture places the nsp $13_{\mathrm{T}}$ active site directly in the path of the downstream template-RNA (t-RNA). The cryo-EM maps showed that the $5^{\prime}$ single-stranded overhang of the t-RNA (Extended Data Fig. 1) passed through the nucleic acid binding channel of nsp $13_{\mathrm{T}}$ (ref. ${ }^{24}$ ), but the low resolution of the map due to structural heterogeneity precluded detailed modeling ${ }^{20}$.

The structural analysis of the nsp $13_{2}-\mathrm{RTC}$ provided new perspectives into the role of the nsp 13 helicase in the complex viral replication-transcription program, suggesting that nsp13 may facilitate processive elongation by the RdRp on the highly structured RNA genome ${ }^{25,26}$, but may also generate backtracked RTCs for proofreading, template-switching during subgenomic RNA transcription or both ${ }^{20,27}$. How nsp 13 directs these diverse processes that regulate RdRp function remains less well understood. For instance, the structures indicate that nsp13 translocates on the t-RNA strand in the $5^{\prime} \rightarrow 3^{\prime}$ direction ${ }^{16}$, while the RdRp would translocate on the same strand in the opposite direction (Extended Data Fig. 1). How can the RdRp rapidly replicate the roughly 30 kilobase $(\mathrm{kb})$ viral genome ${ }^{28}$ if it is opposed by the helicase? Also, what is the role of the second nsp13 protomer (nsp13 $3_{\mathrm{F}}$ ), which appears capable of ATPase and translocation/helicase activity but does not appear to be engaged with nucleic acid in the structures ${ }^{20,27}$ ?

Here we describe an extensive structural analysis of a cryo-EM dataset of the nsp13-RTC, combined with molecular dynamics (MD) simulation analysis of the resulting structures. The results yield a cryo-EM map of the nsp $13_{2}-\mathrm{RTC}$ at a nominal resolution of $2.8 \AA$ (2.1-2.5 $\AA$ in the active site core of the RdRp, Extended Data Figs. 2-4). Structural heterogeneity apparent in the nsp13 portions of the map was resolved by classification approaches, revealing four

'Laboratory of Molecular Biophysics, The Rockefeller University, New York, NY, USA. ${ }^{2}$ D. E. Shaw Research, New York, NY, USA. ${ }^{3}$ The National

Resource for Automated Molecular Microscopy, Simons Electron Microscopy Center, New York Structural Biology Center, New York, NY, USA. ${ }^{4}$ Gilead Sciences, Inc., Foster City, CA, USA. ${ }^{5}$ Department of Biochemistry and Molecular Biophysics, Columbia University, New York, NY, USA. ${ }^{6}$ These authors contributed equally: James Chen, Qi Wang, Brandon Malone. ${ }^{7}$ These authors jointly supervised this work: David E. Shaw, Seth A. Darst.

凶e-mail: David.Shaw@DEShawResearch.com; darst@rockefeller.edu 
distinct conformational states of the nsp13 subunits. Analysis of these conformational states suggest solutions to the apparent contradictions regarding the role of nsp 13 and provides further insight into models for nsp13 function during viral replication-transcription.

\section{Results}

An augmented cryo-EM dataset allows nsp13-RTC structural analysis. Previously we described a single-particle cryo-EM analysis of a stable SARS-CoV-2 nsp13-RTC from a curated set of 88,058 particle images $^{20}$. These particles were classified into three distinct assemblies, nsp13 ${ }_{1}$-RTC (4.0 ̊ nominal resolution), nsp13 $3_{2}-\mathrm{RTC}$ $(3.5 \AA)$ and a dimer of nsp $13_{2}-$ RTC $\left(\left(\text { nsp } 13_{2}-\text { RTC }\right)_{2} ; 7.9 \AA\right)$. Here we analyzed a much larger dataset (nearly five times as many particles, Extended Data Fig. 2 and Supplementary Table 1) collected from the same sample preparation. From a consensus refinement (Extended Data Figs. 2 and 3, map 1; note that Extended Data Fig. 2 shows the details of the cryo-EM processing pipeline and Extended Data Fig. 3 is a streamlined cryo-EM processing pipeline that highlights the essential steps), the particles were classified ${ }^{29}$ into the same three assemblies observed previously (nsp13 $3_{1}$ RTC (map 2), nsp $13_{2}-$ RTC (map 3), (nsp13 ${ }_{2}$ RTC) ${ }_{2}($ map 4$\left.)\right)^{20}$ with a very similar distribution of particles between the three assemblies (Table 1, Extended Data Figs. 2 and 3 and Supplementary Table 1), confirming the robustness of the classification procedure. We focus primarily on the nsp $13_{2}-$ RTC because the bulk of the particles (72\%) belong to this class and generated the highest resolution map (Extended Data Figs. 2-4, map 3; 2.9 Å nominal resolution).

To obtain the best possible consensus cryo-EM map of the entire complex, we generated a series of cryo-EM maps by focused refinement around subdomains of the nsp13 $13_{1}$ RTC (map 2) and nsp13 ${ }_{2}$-RTC (map 3) maps and combined these, generating a composite map with a nominal resolution of $2.8 \AA$ (Extended Data Figs. 2-4, map9). Local-resolution analysis ${ }^{30}$ suggested that the active site and the nidovirus RdRp-associated nucleotidyl transferase domain (NiRAN) ligand-binding pocket of the RdRp were resolved to between 2.1 and $2.6 \AA$ resolution (Extended Data Fig. 4). This was supported by the excellent quality of the cryo-EM map, where the ADP- $\mathrm{Mg}^{2+}$ bound in the NiRAN domain enzymatic site could be visualized (Extended Data Fig. 5) and RNA base pairs near the RdRp active site could be identified directly from the cryo-EM density (Extended Data Fig. 5). Although not as well resolved, the $\mathrm{ADP}-\mathrm{AlF}_{3}-\mathrm{Mg}^{2+}$ and surrounding residues in the nsp13 active sites could also be modeled (Extended Data Fig. 5).

Despite the excellent map quality for most of the RTC (Extended Data Figs. 1 and 4), features of the composite consensus map (map9) suggested substantial heterogeneity in the nsp13 subunits, particularly in the RecA2 and $1 \mathrm{~B}$ domains (Extended Data Fig. 4). Therefore, we generated a mask surrounding the $n s p 13_{\mathrm{T}}$ and $n s p 13_{\mathrm{F}}$ RecA1, RecA2 and $1 \mathrm{~B}$ domains (of map 3, Fig. 1 and Extended Data Fig. 3) and used masked classification with signal subtraction ${ }^{31}$ to identify four distinct conformational states (Table 2, Fig. 1 and Extended Data Figs. 2, 3 and 6) with substantial differences in the dispositions of the nsp13 subunits, particularly nsp $13_{\mathrm{T}}$.

The class II structure (Fig. 1 and Extended Data Fig. 6) contains the most particles and the nsp13 subunits are best resolved in this map (map 12, Fig. 1 and Extended Data Figs. 3 and 6). Compared to the other structures, the nsp13 ${ }_{\mathrm{T}}$ RecA domains of class II (map 12) are closed onto each other more than the other structures (Extended Data Fig. 6) and are thereby engaged most tightly with the RNA (below). We call this the 'nsp13 $3_{\mathrm{T}}$-engaged' structure and use it as a reference to give a general overview of the conformational changes in the other classes.

While each of the classes shows substantial changes in both the disposition of each nsp13 subunit as a whole as well as intramolecular domain motions within each nsp13 subunit, each
Table 1 | Cryo-EM data collection, refinement and validation statistics for nsp13,-RTC, nsp13 - RTC and (nsp13 - RTC)

$\begin{array}{lll}\text { nsp13,-RTC } & \begin{array}{l}\text { nsp13 }- \text { RTC } \\ \text { (EMDB- }\end{array} & \begin{array}{l}\text { (composite) } \\ \text { (nTC) })_{2}-\end{array} \\ \text { 24431) } & \text { (EMDB-24430) } & \text { (EMDB- } \\ \text { (PDB 7RE2) } & \text { (PDB 7RE1) } & \text { 24432) } \\ & & \text { (PDB 7RE3) }\end{array}$

Data collection and processing

\begin{tabular}{|c|c|c|c|}
\hline Magnification & \multicolumn{3}{|l|}{81,000} \\
\hline Voltage (kV) & \multicolumn{3}{|l|}{300} \\
\hline $\begin{array}{l}\text { Electron exposure } \\
\left(\mathrm{e}^{-} / \AA^{2}\right)\end{array}$ & \multicolumn{3}{|l|}{66} \\
\hline Defocus range $(\mu \mathrm{m})$ & \multicolumn{3}{|c|}{-0.8 to -2.5} \\
\hline Pixel size $(\AA)$ & \multicolumn{3}{|c|}{1.07} \\
\hline Symmetry imposed & \multicolumn{3}{|l|}{$\mathrm{C} 1$} \\
\hline $\begin{array}{l}\text { Initial particle images } \\
\text { (no.) }\end{array}$ & \multicolumn{3}{|c|}{$3,750,107$} \\
\hline $\begin{array}{l}\text { Final particle images } \\
\text { (no.) }\end{array}$ & 85,187 & 315,120 & 35,392 \\
\hline $\begin{array}{l}\text { Map resolution }(\AA) \\
\text { FSC threshold } 0.143\end{array}$ & 3.2 & 2.8 & 3.3 \\
\hline $\begin{array}{l}\text { Map resolution } \\
\text { range }(\AA)\end{array}$ & $2.7-6.2$ & $2.1-6.7$ & $2.9-6.4$ \\
\hline
\end{tabular}

Refinement

Initial models used (PDB code)

6XEZ, 6YYT, 6XEZ, 6YYT, 6ZSL 6ZSL 6ZSL

Model resolution $(\AA)$

3.2

2.8

6XEZ, 6YYT,

FSC threshold 0.5

Map sharpening

$-81.9$

$-61.1$

$-97.4$

$B$ factor $\left(\AA^{2}\right)$

Model composition

Nonhydrogen atoms

17,096

21,825

43,154

Protein residues

1,963

2,553

5,106

Nucleic acid residues

71

71

142

(RNA)

Ligands

13

19

34

$B$ factors $\left(\AA^{2}\right)$

Protein

Nucleic acids

67.76

88.73

151.00

Ligand

151.77

175.13

158.63

76.87

77.6

200.51

R.m.s. deviations

Bond lengths ( $\AA$ )

0.005

0.004

0.004

Bond angles $\left(^{\circ}\right)$

0.591

0.655

0.997

Validation

\begin{tabular}{llll} 
MolProbity score & 2.11 & 2.54 & 2.60 \\
Clashscore & 8.79 & 7.84 & 10.86 \\
\hline Poor rotamers (\%) & 2.42 & 8.64 & 6.67 \\
Ramachandran plot & & & \\
Favored (\%) & 94.98 & 94.02 & 93.68 \\
Allowed (\%) & 5.02 & 5.98 & 6.32 \\
Disallowed (\%) & 0 & 0 & 0
\end{tabular}

structural class can be characterized by one dominant conformational change in $n s p 13_{\mathrm{T}}$ (compared to the $\mathrm{nsp} 13_{\mathrm{T}}$-engaged structure used as a reference): 


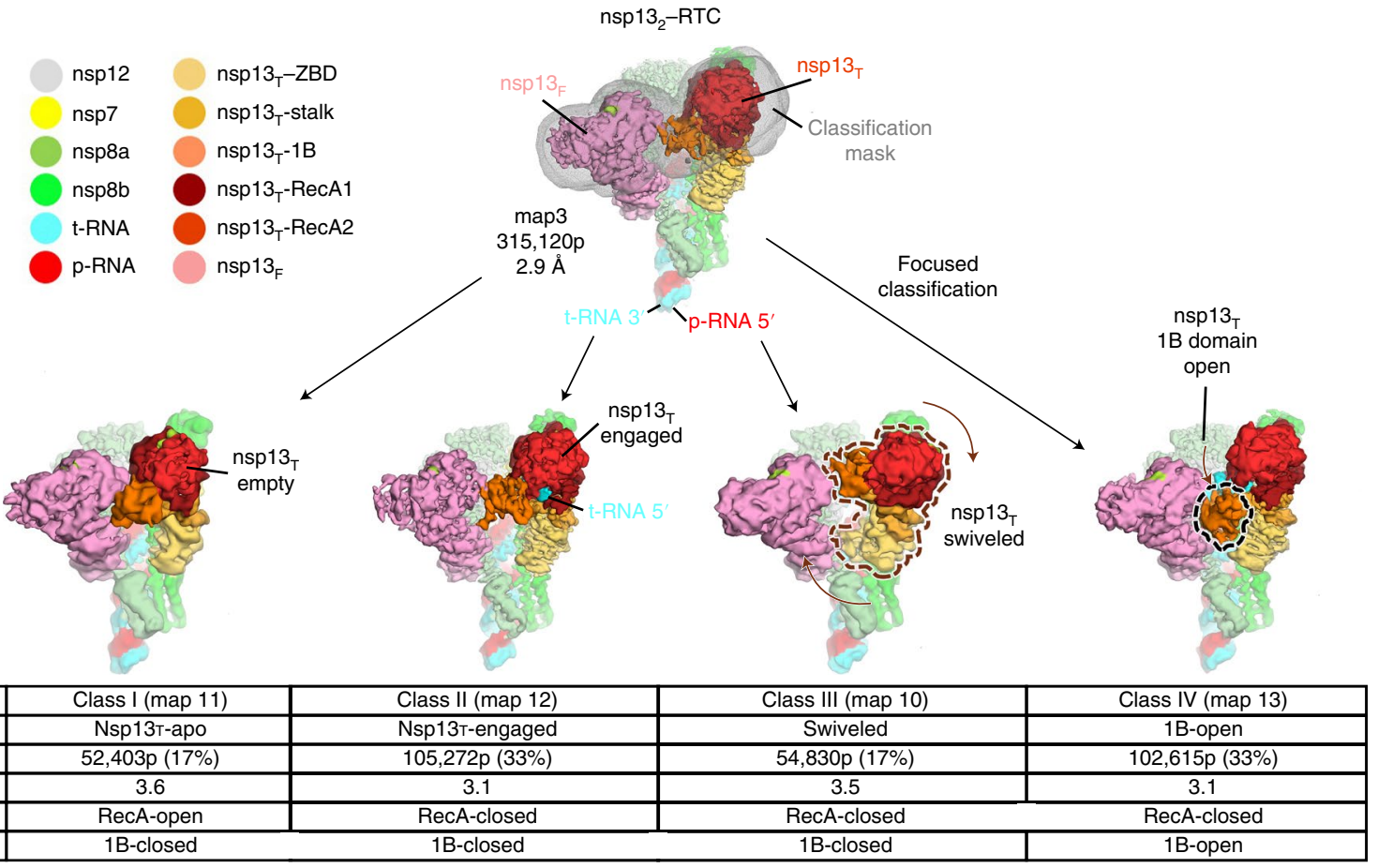

Fig. 1 | Four conformational states of the nsp13 -RTC. Top, cryo-EM density (map 3, local-resolution filtered) colored according to the code on the left. A mask was constructed surrounding the $n s p 13_{T}$ and nsp13 $1 \mathrm{~B}$, RecA1 and RecA2 domains (gray mesh). The 315,120 particles were divided into four distinct structures (classes I-IV) by focused classification inside the mask, followed by further refinement (Extended Data Figs. 2 and 6 ). Class II contained the most particles, and the nsp13 $\operatorname{RecA}$ domains were completely closed (Extended Data Fig. 6), entrapping the $5^{\prime} \mathrm{t}$-RNA segment in a groove between the two RecA domains and the 1B domain (Fig. 2). Therefore, class II (nsp13 $T_{T}$-engaged) was used as a reference for comparison of the other structures. Each class was characterized by one dominant conformational change (class I) nsp13 ${ }_{\mathrm{T}}$-apo, the RecA domains were completely open (Extended Data Fig. 6 ) and devoid of RNA (Fig. 3), (class III) swiveled, the nsp13 protomer as a whole was rotated $38^{\circ}$ as shown (Fig. 5), (class IV) 1B-open, the nsp13 1 1B domain was rotated open by $85^{\circ}$ (Fig. 4) (Extended Data Fig. 6 and Supplementary Videos 1 and 2).

(1) In class $I$, the $n s p 13_{\mathrm{T}}$ RecA2 domain is rotated open by $21^{\circ}$ with respect to RecA1. Concomitantly, the RNA-binding site is empty while occupancy of the nsp $13_{\mathrm{T}}$ nucleotide-binding site is ambiguous. We therefore call this the 'nsp $13_{\mathrm{T}}$-apo' structure (Fig. 1).

(2) In class III, the nsp13 subunit swivels as a whole by $38^{\circ}$ away from nsp $13_{\mathrm{F}}$. We call this the 'nsp13 ${ }_{\mathrm{T}}$-swiveled' structure (Fig. 1).

(3) In class IV, the nsp $13_{\mathrm{T}}$ domain $1 \mathrm{~B}$ is rotated $85^{\circ}$ away from the nsp13 ${ }_{\mathrm{T}}$ RNA-binding channel, creating the '1B-open' structure (Fig. 1).

The nsp $13_{\mathrm{T}}$-engaged conformation grasps the downstream RNA t-strand. In the nsp $13_{\mathrm{T}}$-engaged structure, the distance between the center-of-gravity of the two nsp $13_{\text {T }}$ RecA domains, $27.3 \AA$, is the shortest of the eight nsp13 conformations (Extended Data Fig. 6). The RecA domains are thus 'closed' and grasp the downstream t-RNA single-stranded 5 ' segment emerging from the RdRp active site, giving rise to well-resolved cryo-EM density for the RNA passing through the helicase (Fig. 2a). The RNA is corralled in a tunnel between the two RecA domains and the $1 \mathrm{~B}$ domain, which is also in a closed conformation (Figs. 1 and 2a). The pattern of purines and pyrimidines in the cryo-EM density is clearly discernable, allowing the unique sequence register of the RNA engaged with the $n s p 13_{T}$ helicase to be determined (Fig. 2a).

The ordered RNA segment is 7 nucleotides (nt) in length (+9 to +15 ; Fig. 2), with the five central nucleotides $(+10$ to +14$)$ completely enclosed within the helicase. The RNA phosphate backbone generally faces the nsp $13_{\mathrm{T}}$ RecA domains and the mostly stacked bases face the 1B domain (Fig. 2). As might be expected, the helicase establishes extensive interactions with the RNA phosphate backbone, including several polar interactions. Interactions with the RNA bases are mostly van der Waals interactions and not expected to be base-specific (Fig. 2).

The nsp $13_{\mathrm{T}}$-apo state. Comparison of the $\mathrm{nsp} 13_{\mathrm{T}}$-apo and nsp $13_{\mathrm{T}}$-engaged structures revealed a striking change in the conformation of the RecA-like ATPase domains of nsp13. Superimposition of the $\alpha$-carbons of nsp13 $3_{\mathrm{T}}$ RecA1 (residues 235-439) or RecA2 (residues 440-596) alone yielded root-mean-square deviations (r.m.s.d.s) of 0.387 and $0.673 \AA$, respectively, indicating the structures of the individual domains are very similar between the two states. However, superimposition via the $\alpha$-carbons of only RecA1 gave an r.m.s.d. of $7.05 \AA$ for the RecA $2 \alpha$-carbons, indicating a substantial change in the relative disposition of the two domains. The movement of RecA2 with respect to RecA1 corresponds to a roughly $21^{\circ}$ rotation about the axis shown in Fig. 3a (Supplementary Video 1), corresponding to an opening of the RecA domains; the nsp13 ${ }_{\mathrm{T}}$ RecA domains of the nsp13 ${ }_{\mathrm{T}}$-apo state are the furthest open of any of the eight nsp13 protomer structures (Extended Data Fig. 6).

The consensus nsp13 $3_{2}$ RTC cryo-EM map (map 3, Extended Data Figs. 2 and 3) contains low-resolution density indicating that the downstream single-stranded $5^{\prime}$ segment of the t-RNA occupies the nsp $13_{\mathrm{T}}$ RNA-binding channel (Extended Data Fig. 1). Moreover, the t-RNA 5 ' segment occupying the nsp $13_{\mathrm{T}}$ RNA-binding channel of the nsp $13_{\mathrm{T}}$-engaged state is well resolved (Fig. 2). By contrast, the nsp $13_{\mathrm{T}}$-apo cryo-EM density shows that the nsp $13_{\mathrm{T}}$ RNA-binding path is empty (Fig. 3b). The nsp $13_{\mathrm{T}}$-apo cryo-EM density also does not support occupancy of $\mathrm{ADP}-\mathrm{AlF}_{3}-\mathrm{Mg}^{2+}$ in the nucleotide-binding 
Table 2 | Cryo-EM data collection, refinement and validation statistics for the nsp13 - RTC conformational states

\begin{tabular}{|c|c|c|c|c|}
\hline & $\begin{array}{l}\text { nsp13.1-apo } \\
\text { (EMDB-24428) } \\
\text { (PDB 7RDZ) }\end{array}$ & $\begin{array}{l}\text { nsp13.1-engaged } \\
\text { (EMDB-24427) } \\
\text { (PDB 7RDY) }\end{array}$ & $\begin{array}{l}\text { nsp13.1-swiveled } \\
\text { (EMDB-24429) } \\
\text { (PDB 7REO) }\end{array}$ & $\begin{array}{l}\text { 1B-open } \\
\text { (EMDB-24426) } \\
\text { (PDB 7RDX) }\end{array}$ \\
\hline \multicolumn{5}{|l|}{ Data collection and processing } \\
\hline Voltage (kV) & 300 & & & \\
\hline Electron exposure $\left(\mathrm{e}^{-} / \AA^{2}\right)$ & 66 & & & \\
\hline Symmetry imposed & $\mathrm{C} 1$ & & & \\
\hline Initial particle images (no.) & 315,120 & & & \\
\hline Final particle images (no.) & 52,403 & 102,615 & 54,830 & 105,272 \\
\hline $\begin{array}{l}\text { Map resolution }(\AA) \\
\text { FSC threshold } 0.143\end{array}$ & 3.6 & 3.1 & 3.5 & 3.1 \\
\hline Initial models used (PDB code) & $6 X E Z, 6 Z S L$ & $6 X E Z, 6 Z S L$ & $6 X E Z, 6 Z S L$ & $6 X E Z, 6 Z S L$ \\
\hline $\begin{array}{l}\text { Model resolution }(\AA) \\
\text { FSC threshold } 0.5\end{array}$ & 3.6 & 3.1 & 3.5 & 3.1 \\
\hline Map sharpening $B$ factor $\left(\AA^{2}\right)$ & -78.3 & -84.3 & -75.8 & -82.3 \\
\hline \multicolumn{5}{|l|}{ Model composition } \\
\hline Nonhydrogen atoms & 21,545 & 21,846 & 21,563 & 21,828 \\
\hline Protein residues & 2,553 & 2,553 & 2,553 & 2,553 \\
\hline Nucleic acid residues (RNA) & 71 & 78 & 71 & 80 \\
\hline Ligands & 13 & 19 & 16 & 19 \\
\hline \multicolumn{5}{|l|}{$B$ factors $\left(\AA^{2}\right)$} \\
\hline \multicolumn{5}{|l|}{ Validation } \\
\hline MolProbity score & 1.70 & 2.68 & 2.60 & 1.82 \\
\hline Clashscore & 6.09 & 8.05 & 12.57 & 6.30 \\
\hline Poor rotamers (\%) & 0.00 & 10.18 & 9.10 & 0.00 \\
\hline \multicolumn{5}{|l|}{ Ramachandran plot } \\
\hline Favored (\%) & 94.76 & 92.40 & 93.01 & 93.66 \\
\hline Allowed (\%) & 5.24 & 7.60 & 6.80 & 6.30 \\
\hline Disallowed (\%) & 0 & 0 & 0.20 & 0.04 \\
\hline
\end{tabular}

site of nsp $13_{\mathrm{T}}$, although the low resolution of the map in this region makes this conclusion tentative.

Spontaneous transition of $n s p 13_{\mathrm{T}}$ between the engaged and apo conformations. To characterize the RecA1-RecA2 interdomain movement and how a bound substrate may influence that movement, we performed MD simulations of free nsp $13_{\mathrm{T}}$ (that is, without nsp $13_{\mathrm{F}}$ or the RTC) under four different substrate-bound conditions (ATP- $\mathrm{Mg}^{2+} / \mathrm{RNA}, \mathrm{ADP}-\mathrm{Mg}^{2+} / \mathrm{RNA}, \mathrm{ATP}-\mathrm{Mg}^{2+}$ only and ADP- $\mathrm{Mg}^{2+}$ only). For each condition, we ran three independent 5- $\mu$ s simulations, all initiated from the nsp $13_{\mathrm{T}}$-engaged conformation (Figs. 1 and 2).
In simulations of ATP- $\mathrm{Mg}^{2+} / \mathrm{RNA}$-bound $\mathrm{nsp} 13_{\mathrm{T}}$, the RecA2 domain maintained its general orientation with respect to RecA1 throughout the simulations (Fig. 3c). The average r.m.s.d. of RecA2 between the initial nsp13 $3_{\mathrm{T}}$-engaged cryo-EM structure and the structures from the MD trajectories, aligned on the RecA1, was low (roughly $2.9 \AA$, some adjustment of RecA2 from the initial nsp13 ${ }_{\mathrm{T}}$-engaged cryo-EM structure position in these simulations was expected, as the cryo-EM structure was determined using $\mathrm{ADP}-\mathrm{AlF}_{3}$ / RNA in place of ATP- $\left.\mathrm{Mg}^{2+} / \mathrm{RNA}\right)$. Conformations resembling the nsp13 ${ }_{\mathrm{T}}$-apo structure (r.m.s.d. $<3.5 \AA$ ) were not observed (Fig. 3c,d).

In simulations of ADP- $\mathrm{Mg}^{2+} / \mathrm{RNA}$-bound $\mathrm{nsp} 13_{\mathrm{T}}$, RecA2 rotated away from its initial position in the nsp $13_{\mathrm{T}}$-engaged conformation 


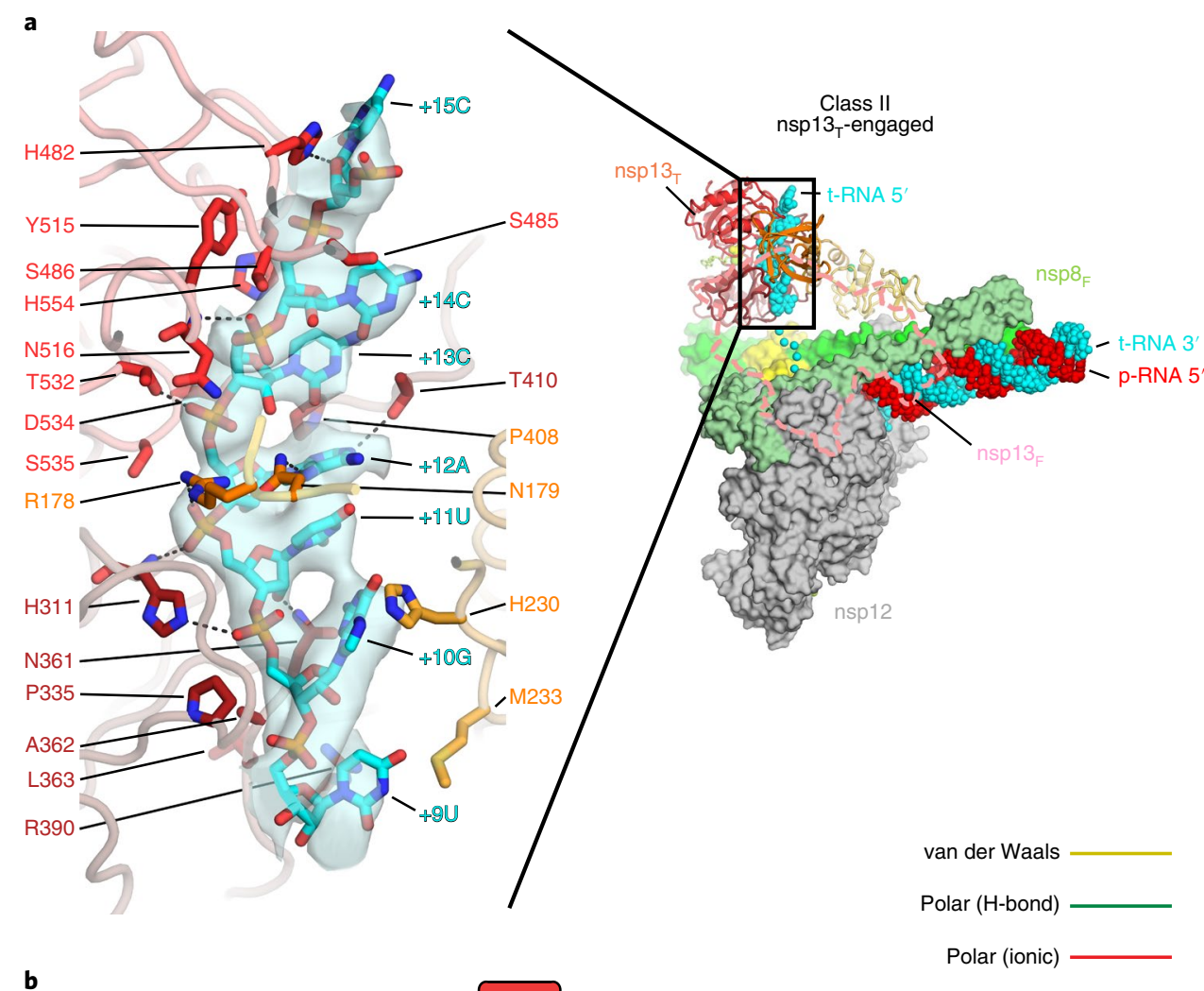

b

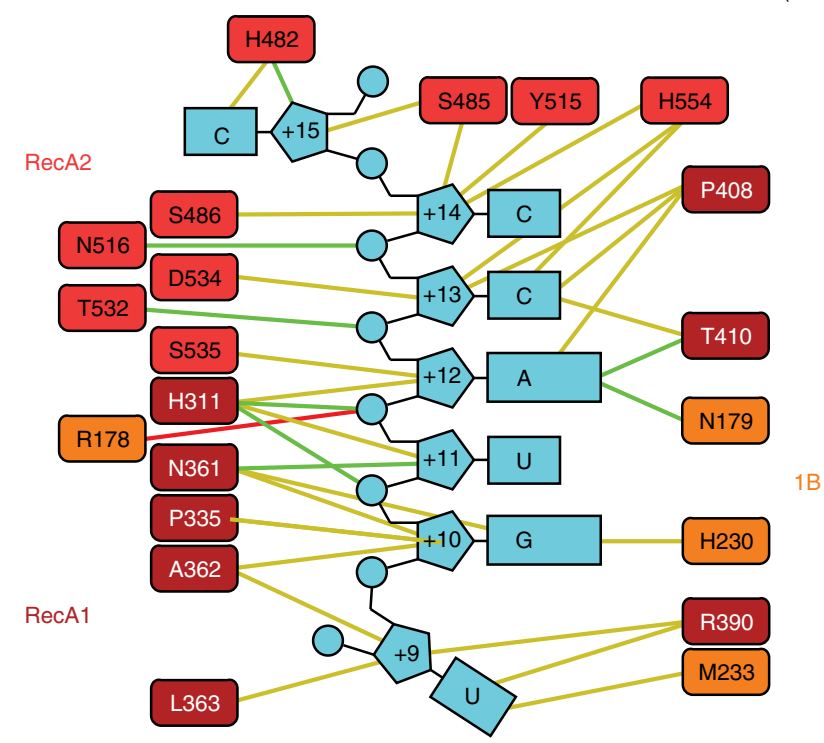

Fig. 2 | In class II (nsp13 ${ }_{T}$-engaged), the nsp13 $3_{\mathrm{T}}$ RecA domains and 1B domain clamp onto the $\mathbf{5}^{\prime}$ single-stranded t-RNA. a, Right, overall view of the $n s p 13_{T}$-engaged structure. Proteins are shown as molecular surfaces except $n s p 13_{T}$ is shown as a backbone ribbon, and nsp13 $3_{\mathrm{F}}$ is removed and shown only as a dashed outline. The RNA is shown as atomic spheres. The boxed region is magnified on the left. Left, nsp13 ${ }_{T}$ is shown as a backbone worm but with side chains that interact with the t-RNA shown. Cryo-EM density for the downstream 5' t-RNA segment is shown (transparent blue surface) with the t-RNA model superimposed. The pattern of purines/pyrimidines in the RNA density was clear and unique, allowing the identification of the sequence register for the nsp13 $3_{T}$-bound RNA. b, Schematic illustrating nsp13 ${ }_{T}$-RNA interactions (Supplementary Video 2).

and $\mathrm{nsp} 13_{\mathrm{T}}$-apo-like conformations were repeatedly visited throughout the simulations (Fig. 3c,d). The ADP- $\mathrm{Mg}^{2+} / \mathrm{RNA}-$ bound nsp $13_{\mathrm{T}}$-apo-like conformations were metastable, and interconverted with the nsp $13_{\mathrm{T}}$-engaged conformations. Spontaneous and reversible conversion between the nsp $13_{\mathrm{T}}$-engaged and $\mathrm{nsp} 13_{\mathrm{T}}$-apo conformations was also observed in the simulations of ATP- $\mathrm{Mg}^{2+}$-bound and $\mathrm{ADP}-\mathrm{Mg}^{2+}$-bound $\mathrm{nsp} 13_{\mathrm{T}}$ (Fig. $3 \mathrm{c}, \mathrm{d}$ ). These results indicate that the presence of both the ATP- $\mathrm{Mg}^{2+}$ and RNA may stabilize the nsp $13_{\mathrm{T}}$-engaged conformation and that the absence of either substrate may destabilize the $\mathrm{nsp} 13_{\mathrm{T}}$-engaged conformation and facilitate the transition to the $\mathrm{nsp} 13_{\mathrm{T}}$-apo conformation, consistent with the observations from the cryo-EM analysis.

The nsp13 '1B-open' conformation explains RNA synthesis in the presence of nsp13. In the nsp13 $3_{\mathrm{T}}$-engaged state, the downstream single-stranded t-RNA is guided through a deep groove between the RecA1 and RecA2 domains that is completely closed off by the $1 \mathrm{~B}$ domain (Fig. 4a). In the 1B-open structure, the nsp13 $1 \mathrm{~B}$ domain 
rotates $85^{\circ}$ about the stalk away from the nsp $13_{\mathrm{T}}$ RNA-binding channel, creating an open groove rather than a closed tunnel (Fig. 4b). The cryo-EM density allows modeling of the downstream single-stranded t-RNA emerging from the RdRp active site up to the edge of the open groove proximal to the RdRp, but the RNA density disappears there, indicating that the RNA is not engaged within the active site of the helicase (Fig. 4b).

In the $1 \mathrm{~B}$-open conformation, the $\mathrm{nsp} 13_{\mathrm{T}} 1 \mathrm{~B}$ domain appears to be trapped open by the presence of nsp13 $3_{\mathrm{F}}$ (Fig. $4 \mathrm{~b}$ ), with the transition from the $1 \mathrm{~B}$-open to the $1 \mathrm{~B}$-closed conformation blocked by nsp $13_{\mathrm{F}}$. Consistent with this, we analyzed the nsp13 conformational states in the nsp13 - RTC (nsp13 $3_{\mathrm{F}}$ absent) by masked classification with signal subtraction around the RecA1, RecA2 and 1B domains of the single nsp13 (Extended Data Fig. 2) but the 1B-open nsp13 conformation was not observed. We propose that the $1 \mathrm{~B}$-open conformation of the $n s p 13_{\mathrm{T}} 1 \mathrm{~B}$ domain is trapped by the presence of nsp 13 .

We note that in the (nsp13 $\left.3_{2}-\mathrm{RTC}\right)_{2}$ dimer (Extended Data Figs. 2-4), the nsp 13 protomers corresponding to $n s p 13_{\mathrm{T}}$ are also in the '1B-open' state, as was observed by Yan et al. ${ }^{32}$. Since the dimer only comprises $8 \%$ of our particle dataset (Supplementary Table 1) while the nsp $13_{2}$-RTC complex comprises $72 \%$ of the particles, we have focused our attention on the latter complex. We observe that the (nsp13 $\left.{ }_{2}-\mathrm{RTC}\right)_{2}$ dimer forms in the absence of additional factors such as nsp10-14 (ref. ${ }^{20}$ ), in contrast to what is reported in Yan et al..$^{32}$.

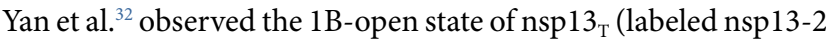
in their nomenclature) in their (dimer) dCap(0)-RTC structure, curiously assigned as a backtracking-competent state. This is at odds with: (1) observations that $n s p 13_{\mathrm{T}}$ in the $1 \mathrm{~B}$-open conformation does not engage RNA in its RNA-binding groove (Fig. 4b, also observed by Yan et al..$^{32}$ ) and so would not be useful for RNA translocation, and (2) the finding that nsp13 stimulated SARS-CoV-2 RTC backtracking ${ }^{27}$.

Spontaneous nsp13 $1 \mathrm{~T}$-open to 1B-closed transition. The conformations of the $\mathrm{nsp} 13_{\mathrm{T}} 1 \mathrm{~B}$ domain in the $\mathrm{nsp} 13_{\mathrm{T}}$-engaged and $\mathrm{nsp} 13_{\mathrm{T}}$-apo structures are related by a roughly $10^{\circ}$ rotation around the nsp13-stalk, but the $1 \mathrm{~B}$ domains are closed on the nsp13-RecA domains in both structures. We refer to these collectively as 1B-closed states (Fig. 1). These conformations have also been observed in crystal structures of isolated nsp13 as well as some other SF1-like helicases ${ }^{33}$. The conformation of the 1B domain in the $1 \mathrm{~B}$-open cryo-EM structure, in which the domain is rotated roughly $85^{\circ}$ compared to the $1 \mathrm{~B}$-closed conformations, was only seen in $n s p 13_{\mathrm{T}}$ when it was paired with nsp $13_{\mathrm{F}}$ in the RTC, suggesting that this conformation may not be stable in isolated nsp13. To test this hypothesis, we performed five independent $25-\mu$ s simulations on isolated (free) nsp13 (with ADP- $\mathrm{Mg}^{2+}$ ), initiated from the 1B-open conformation (Fig. 1).

In three out of the five simulations, the $1 \mathrm{~B}$ domain underwent a roughly $90^{\circ}$ rotation from the starting $1 \mathrm{~B}$-open conformation around the stalk toward the RNA-binding groove to a 1B-closed conformation (Fig. 5a). These roughly $90^{\circ}$ rotated $1 \mathrm{~B}$ domain conformations closely resemble the disposition of the $1 \mathrm{~B}$ domain in the $n s p 13_{\mathrm{T}}$-apo structure. The $1 \mathrm{~B}$ domain r.m.s.d. between the simulation-generated structures from the last $2 \mu$ s of the three trajectories and the $1 \mathrm{~B}$ domain in the $\mathrm{nsp} 13_{\mathrm{T}}$-apo cryo-EM structure (aligned on the RecA1 domain) was, on average, roughly $3.6 \AA$. We also observed that a small portion $(<5 \%)$ of these $1 \mathrm{~B}$-closed structures were more similar to the $1 \mathrm{~B}$ domain of the $n s p 13_{\mathrm{T}}$-engaged conformation (r.m.s.d.<3.5 ). These nsp13 ${ }_{\mathrm{T}}$-engaged-like $1 \mathrm{~B}$ conformations were short-lived, and once visited they quickly transitioned to the nsp $13_{\mathrm{T}}$-apo conformation, presumably because the nsp $13_{\mathrm{T}}$-engaged conformation was captured in the presence of RNA, which was not included in our simulations.

We next asked whether or not the $1 \mathrm{~B}$ domain in a $1 \mathrm{~B}$-closed state may spontaneously transition to the $1 \mathrm{~B}$-open state. In each of the three simulations in which we observed a transition of the $1 \mathrm{~B}$ domain from the $1 \mathrm{~B}$-open to a $1 \mathrm{~B}$-closed conformation, the $1 \mathrm{~B}$ domain remained in the $1 \mathrm{~B}$-closed conformation through the end of the 25- $\mu$ s simulation; a 1B-closed-to-open transition was not observed. We performed an additional five independent $25-\mu$ s simulations of the isolated (free) nsp $13_{\mathrm{T}}$, initiated from the $1 \mathrm{~B}$-closed conformation (of the nsp $13_{\mathrm{T}}$-engaged structure). We did not observe any transition events to the $1 \mathrm{~B}$-open conformation over the aggregated $125-\mu$ s simulation time. Instead, the $1 \mathrm{~B}$ domain maintained its $1 \mathrm{~B}$-closed orientation in the initial structure, with some minor wobbling back and forth between the 1B-closed conformations of the nsp $13_{\mathrm{T}}$-engaged and nsp $13_{\mathrm{T}}$-apo structures (Fig. 5b).

Aligning the $n s p 13_{\mathrm{T}}$ simulation structures in the 1B-open-toclosed transition pathways with the nsp $13_{\mathrm{T}}$ of the $1 \mathrm{~B}$-open cryo-EM structure showed that, on average, roughly $40 \%$ (53\%, 22\% and $45 \%$ in the three simulations) of these $1 \mathrm{~B}$ domain intermediate conformations clashed with nsp13 $3_{\mathrm{F}}$ suggesting that the $1 \mathrm{~B}$-open-to-closed transition might be blocked by $n s p 13_{F}$ (Extended Data Fig. 7). Consistent with this notion, in five independent $25-\mu$ s simulations of nsp $13_{\mathrm{T}}$ in complex with nsp $13_{\mathrm{F}}$ and the RTC (that is, the nsp $13_{2}-$ RTC complex), with nsp $13_{\mathrm{T}}$ initiated from the $1 \mathrm{~B}$-open conformation, we did not observe any transition events to the 1B-closed conformation over the aggregate $125 \mu$ s of simulation time (Fig. 5a). Here we envisage that $1 \mathrm{~B}$ domain transitions are facilitated by entry into the 'swiveled' state. The swiveled structure is characterized by one dominant conformational change; compared to the nsp $13_{\mathrm{T}}$-engaged structure, the nsp $13_{\mathrm{T}}$ protomer as a whole swivels with respect to the rest of the RTC by $38^{\circ}$, repositioning $n s p 13_{\mathrm{T}}$ with respect to $n s p 13_{\mathrm{F}}$ (Fig. $5 \mathrm{c}$ ).

There are some clashes between $n s p 13_{\mathrm{T}}$ and $n s p 13_{\mathrm{F}}$ when the simulation-generated structures are aligned to the swiveled cryo-EM structure, but to a much lesser extent $(9 \%, 0 \%$ and $2 \%$ in the three simulations, Extended Data Fig. 7). This observation is consistent with the notion that the swiveled structure may be an intermediate state that facilitates the transition between the 1B-open and $1 \mathrm{~B}$-closed conformations.

In summary, our simulations suggest that the conformation of the $1 \mathrm{~B}$ domain in the $1 \mathrm{~B}$-open structure may only be transiently stable on its own, transitioning spontaneously into the 1B-closed conformations of the $n s p 13_{\mathrm{T}}$-apo and $n s p 13_{\mathrm{T}}$-engaged structures. Such transitions may be blocked by the presence of nsp $13_{F}$ in the 1B-open nsp13 $12_{2}$ RTC. We did not observe transitions from the

Fig. 3 | In nsp13 ${ }_{\mathrm{T}}$-apo, the RecA domains are open and devoid of RNA density. a, The RecA1 domains of class I (nsp13 ${ }_{\mathrm{T}}$-apo) and class II (nsp13 ${ }_{\mathrm{T}}$-engaged) were superimposed (r.m.s.d. of $0.387 \AA$ over $205 \alpha$-carbons), revealing that the RecA 2 domain of nsp 13 -apo was rotated open by $21^{\circ}$ about the rotation axis shown. The ADP-AIF ${ }_{3}-\mathrm{Mg}^{2+}$ from the nsp13 ${ }_{\mathrm{T}}$-engaged structure is shown as yellow atomic spheres. $\mathbf{b}$, Right, overall view of the nsp13 ${ }_{T}$-apo structure. Proteins are shown as molecular surfaces except $n s p 13_{T}$, which is shown as a backbone ribbon, and nsp13 ${ }_{\mathrm{F}}$ which is removed and shown only as a dashed outline. The RNA is shown as atomic spheres. The boxed region is magnified on the left. Left, cryo-EM density of nsp13 ${ }_{\mathrm{T}}$-apo reveals that the RNA path is

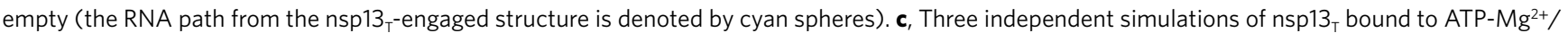
RNA, ADP-Mg ${ }^{2+} /$ RNA, ATP-Mg ${ }^{2+}$ and ADP-Mg2+. Values of r.m.s.d. plotted represent the heavy-atom r.m.s.d. of the RecA2 lobe (after alignment on the

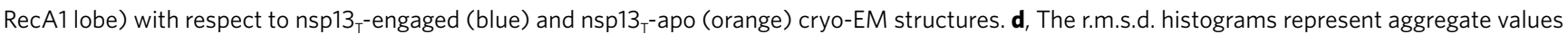
across all three replicates shown in c (Supplementary Video 1). 


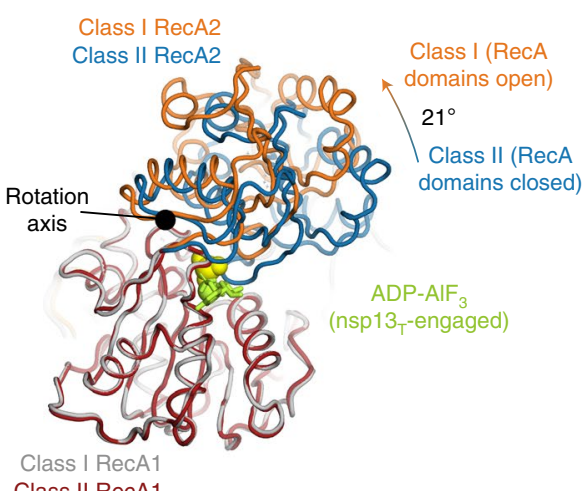

b

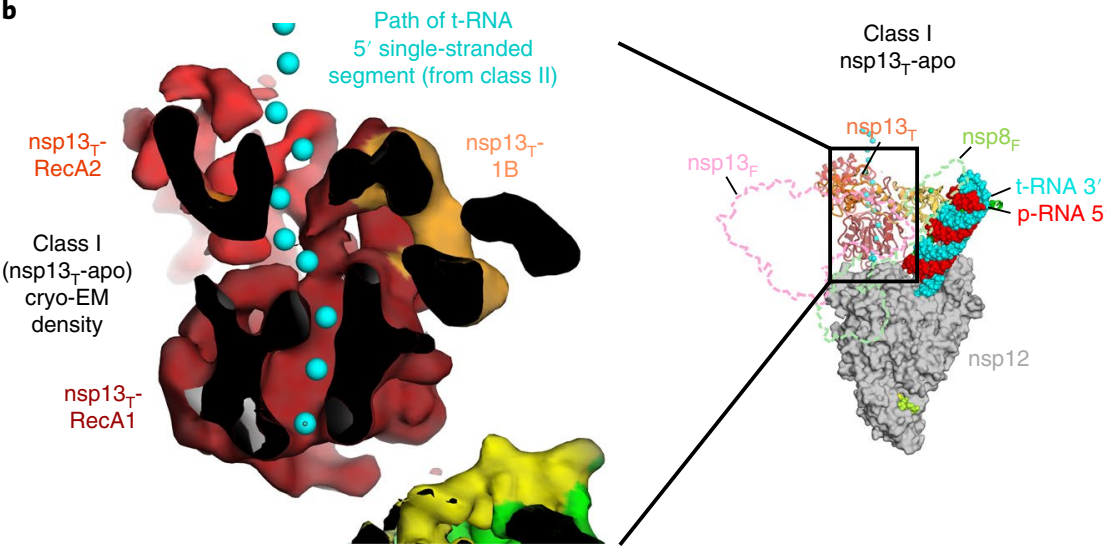

c
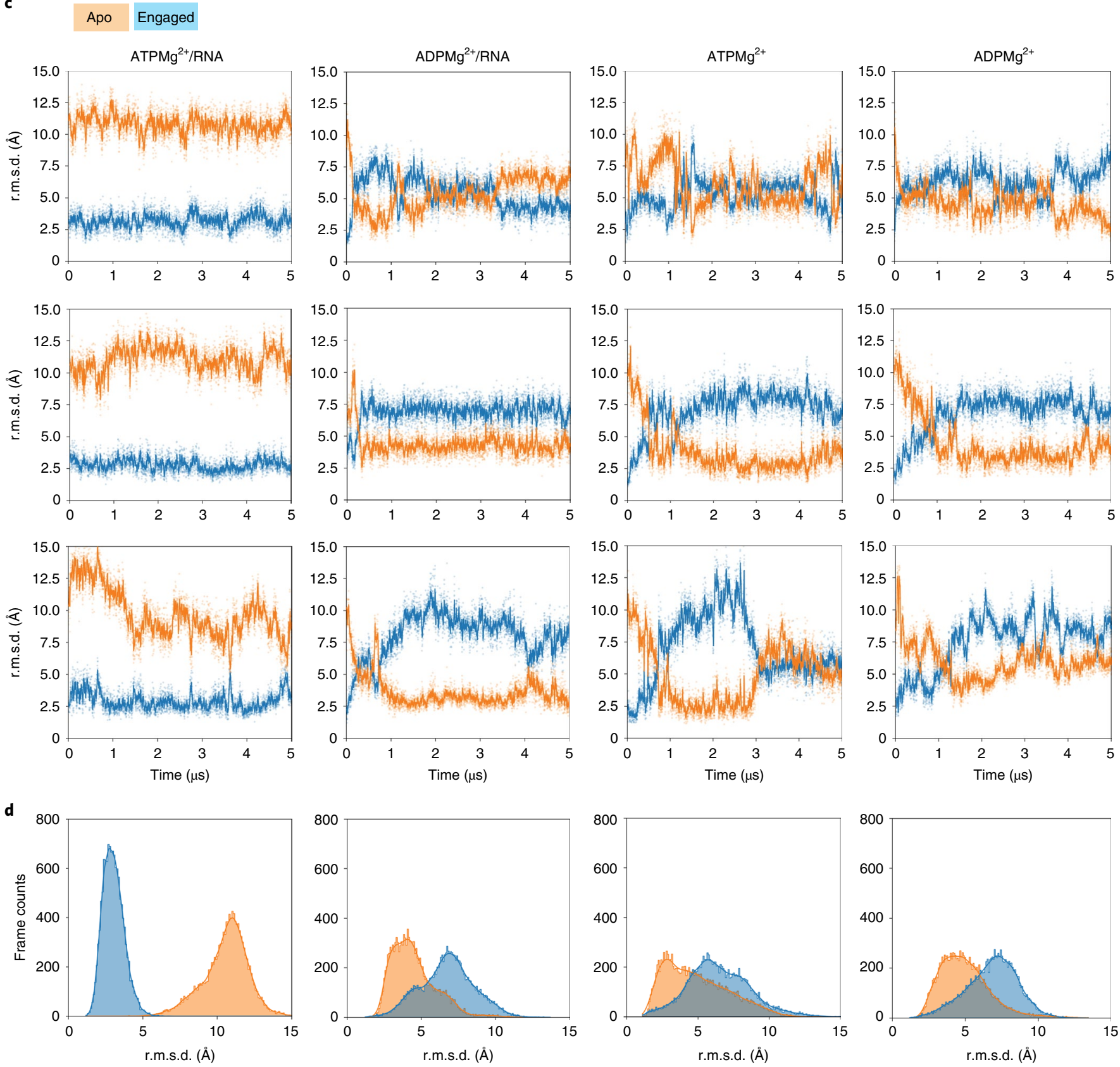

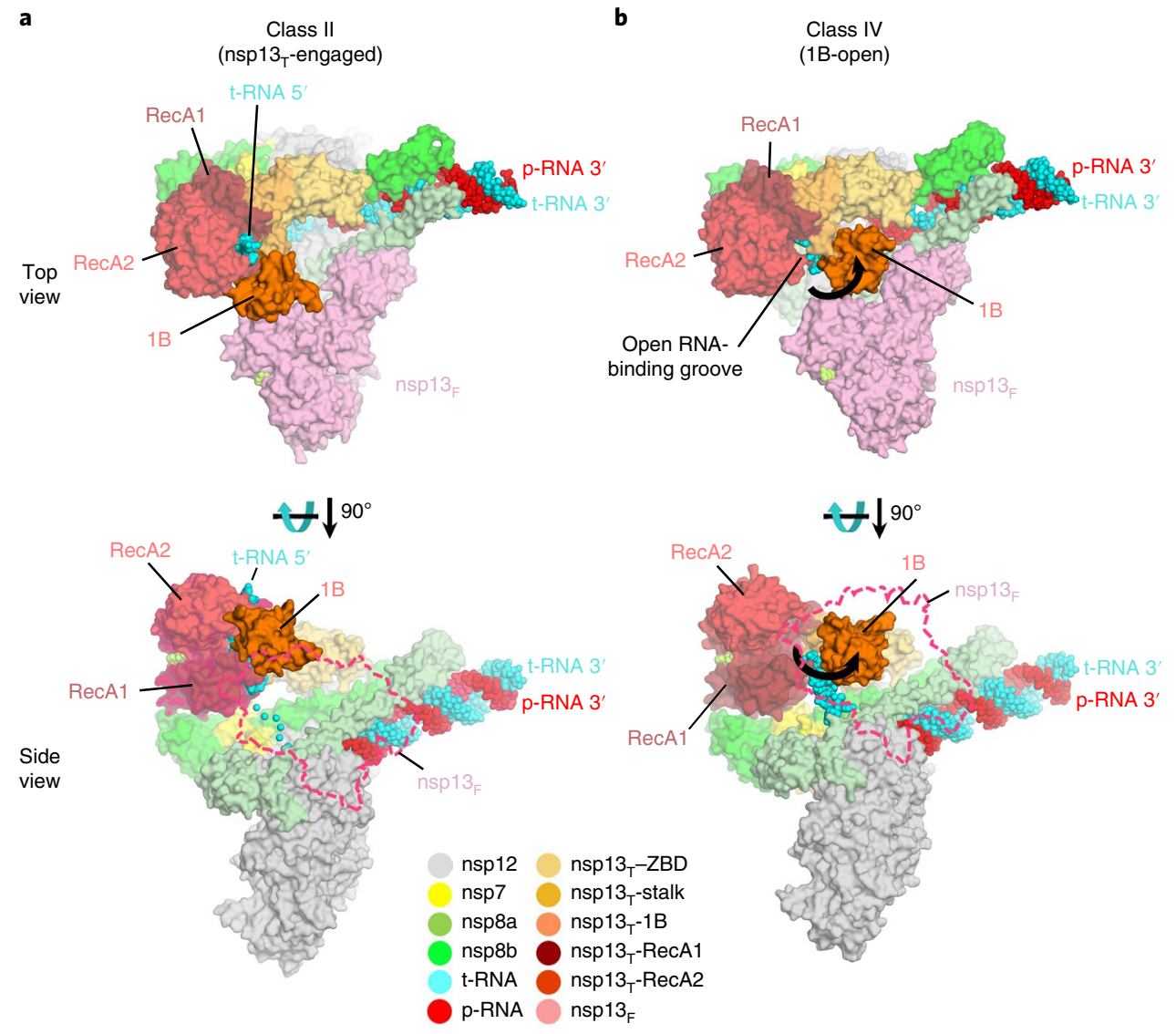

Fig. 4 | 1B-open structure. a,b, Comparison of nsp13 ${ }_{T}$-engaged (a) and 1B-open (b) structures. Two views are shown, a top view (top) and a side view (bottom). In the top view, the proteins are shown as molecular surfaces and color-coded according to the key at the bottom. In the side view, nsp13, is shown only as a dashed outline. The RNA is shown as atomic spheres. In the $1 \mathrm{~B}$-open structure (b), the nsp13 $1 \mathrm{~B}$ domain is rotated open by $85^{\circ}$ (represented by thick black arrows). The 5' t-RNA emerging from the RdRp active site approaches the nsp13, RNA-binding groove but does not enter it (Supplementary Video 2).

1B-closed conformations to the 1B-open conformation, and we speculate that in the presence of RNA in the nsp $13_{\mathrm{T}}$ RNA-binding groove (Fig. 2), nsp13 $3_{\mathrm{T}}$ may be further stabilized in the closed $1 \mathrm{~B}$ domain conformation.

Nsp13 conformations in nsp13 ${ }_{2}$-backtracked complexes. In the nsp13 $3_{\mathrm{T}}$-engaged state (Fig. 1), the RdRp translocates in the $3^{\prime} \rightarrow 5^{\prime}$ direction on the t-RNA while nsp13 $3_{\mathrm{T}}$ grasps the single-stranded t-RNA ahead of the RdRp (Fig. 2) and translocates in the $5^{\prime} \rightarrow 3^{\prime}$ direction (Extended Data Fig. 1). We proposed that events at the RdRp active site that would delay or stall product-RNA ( $\mathrm{p}$-RNA) chain elongation (such as misincorporation or incorporation of nucleotide analogs) could allow the nsp $13_{\mathrm{T}}$ translocation activity to push the RdRp backward on the t-RNA ${ }^{20}$. In this process, termed backtracking, the complex moves in the $5^{\prime} \rightarrow 3^{\prime}$ direction on the t-RNA accompanied by reverse-threading of the p-RNA through the complex, generating a single-stranded p-RNA $3^{\prime}$ fragment. In support of this hypothesis, structural and functional studies showed that the SARS-CoV-2 RdRp can backtrack, that the resulting single-stranded p-RNA $3^{\prime}$ fragment extrudes from the RdRp NTP-entry tunnel and that backtracking is stimulated by nsp13 (ref. ${ }^{27}$ ).

To compare the conformational states of the nsp 13 protomers in the nsp13 $3_{2}$-BTCs (backtracked complexes) with the nsp13 $3_{2}$-RTCs, we used the same masked classification with signal subtraction protocol (Extended Data Fig. 3) to classify the nsp13 $3_{2}$-BTC particles into four conformational states (Extended Data Fig. 8). Structural models were built and rigid-body refined into the cryo-EM densities for each class except for nsp13 ${ }_{2}$-BTC-class 2 ( $13 \%$ of the particles), which had very poor cryo-EM density for $n s p 13_{\mathrm{T}}$. To compare these structural models with the nsp $13_{2}-\mathrm{RTC}$ structures, we aligned the models for each nsp $13_{2}$-BTC model with the nsp $13_{\mathrm{T}}$-engaged state by superimposing $\alpha$-carbons of nsp12, yielding r.m.s.d.s $<0.213 \AA$. We then calculated r.m.s.d.s for $\alpha$-carbons of nsp13 $3_{\mathrm{T}}$ and $n s p 13_{\mathrm{F}}$. Both nsp13 $3_{2}$-BTC-class 1 and nsp13 ${ }_{2}$-BTC-class4 aligned well with the nsp $13_{\mathrm{T}}$-engaged nsp $13_{2}$-RTC state (Supplementary Table 2) and both also had strong density for the downstream $t$-RNA engaged with nsp $13_{\mathrm{T}}$ (Extended Data Fig. 8). Therefore, we classify both of these structures as nsp $13_{\mathrm{T}}$-engaged-BTCs. The nsp $13_{2}$-BTC-class 3 structure had an open $1 \mathrm{~B}$ domain of nsp $13_{\mathrm{T}}$ and clearly aligned with the 1B-open-RTC structure (Extended Data Table 2). Thus, in contrast to the nsp $13_{2}-\mathrm{RTC}$ structures, which were equally divided between

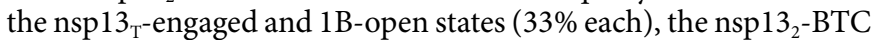
structures were heavily skewed toward the nsp $13_{\mathrm{T}}$-engaged state (72\%) versus the 1B-open state (15\%, Extended Data Fig. 8).

\section{Discussion}

In this work, we observed distinct conformational states of the nsp13 protomers within the SARS-CoV-2 nsp $13_{2}-\mathrm{RTC}$, providing functional insights into nsp13 and its complex with the RTC (Supplementary Video 2). Like other helicases, nsp13 is a molecular motor that translocates along single-stranded nucleic acid, unwinding structural elements in its path ${ }^{16}$. This process is driven by conformational changes within nsp13 resulting from NTP hydrolysis. 


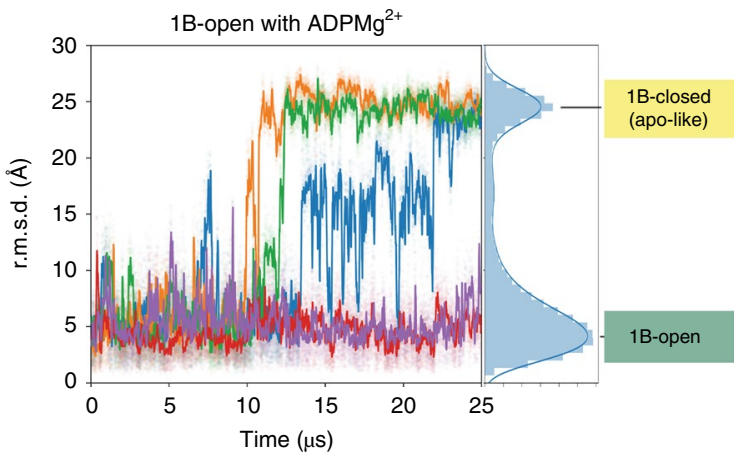

b

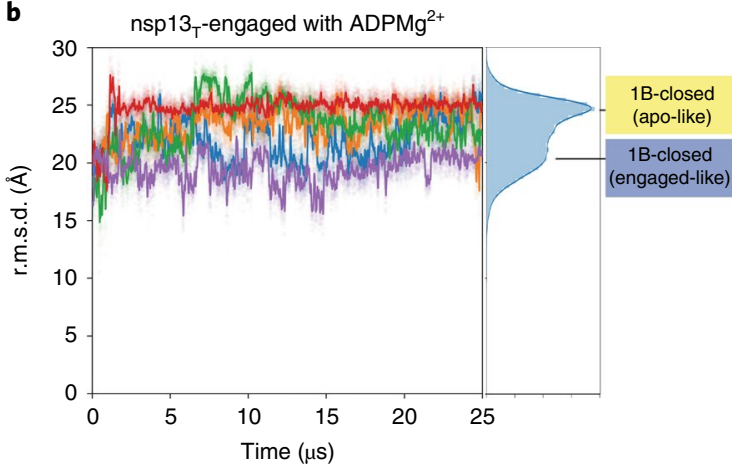

1B-open with $\mathrm{ADPMg}{ }^{2+}$ in the presence of $n s p 13_{F}$ and RTC
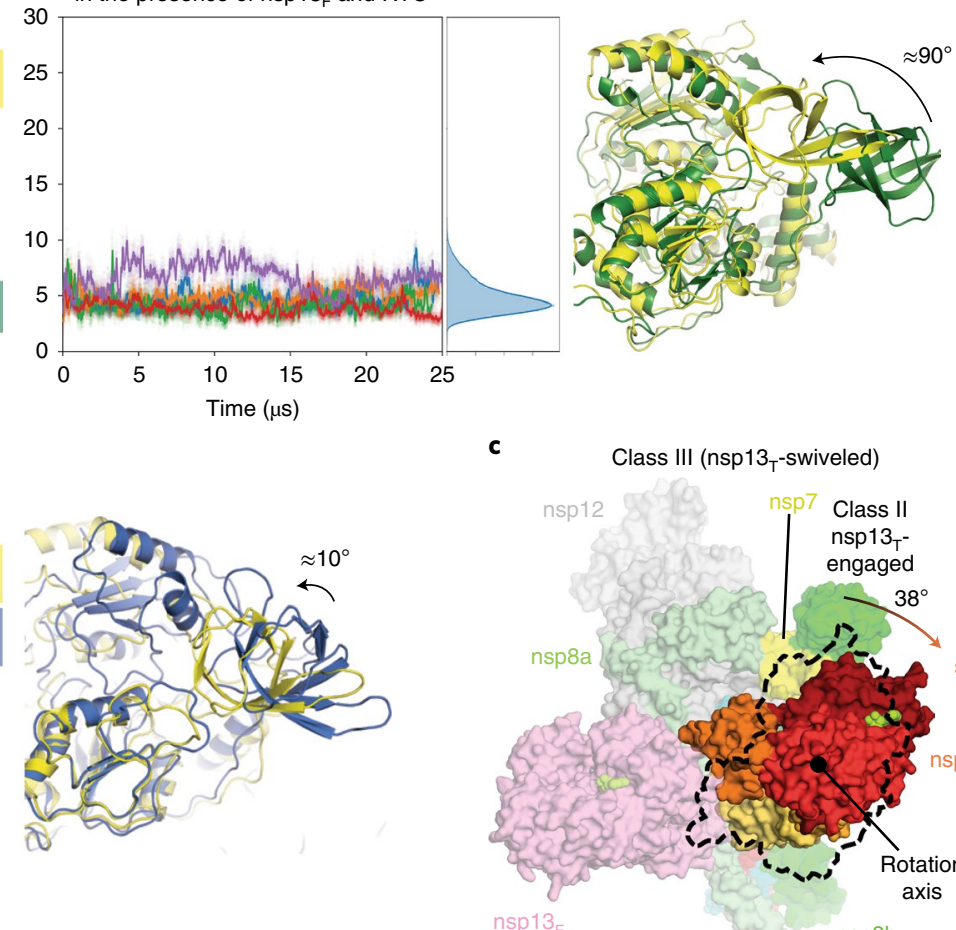

C

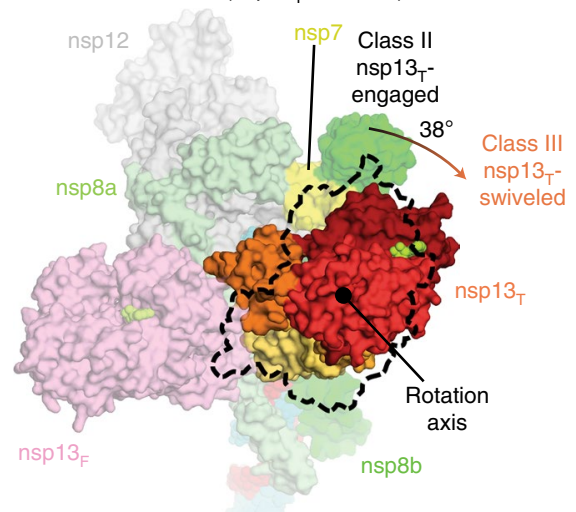

Fig. 5 | In the nsp13 ${ }_{T}$-swiveled structure, the entire nsp13 ${ }_{T}$ promoter is rotated. $\mathbf{a}$, Ten independent simulations of ADP-Mg ${ }^{2+}-$ bound nsp13 ${ }_{T}$, starting from the 1B-open cryo-EM structure, in isolation (five simulations, left-hand plot) and as part of the nsp $13_{2}-$ RTC complex (five simulations, right-hand plot). Values plotted represent the heavy-atom r.m.s.d. of the 1B domain (nsp13 residues 150-228) compared to the 1B domain in the 1B-open cryo-EM structure (aligned on the RecA1 domain). The r.m.s.d. histograms on the right of each plot represent aggregate values across all five simulations. Representative structures of the two major conformations from simulations are shown (right, colored according to the histogram labels). b, Five independent simulations of ADP-Mg2+-bound nsp13 ${ }_{T}$, starting from the nsp13 ${ }_{T}$-engaged state. Values plotted represent the heavy-atom r.m.s.d. of the $1 \mathrm{~B}$ domain compared to the 1B domain in the 1B-open cryo-EM state (aligned on the RecA1 domain). The r.m.s.d. histograms on the far right represent aggregate values across all five simulations. Representative structures of the two major conformations from the r.m.s.d. histogram from simulations are shown (right). c, Front view of the nsp13 $3_{\mathrm{T}}$-swiveled structure, highlighting nsp13 $\mathrm{T}_{\mathrm{T}}$. The position of the $\mathrm{nsp} 13_{\mathrm{T}}$ promoter in the $n s p 13_{\mathrm{T}}$-engaged structure is illustrated by the dashed black

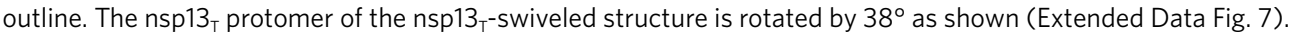

The conformational transition from the nsp $13_{\mathrm{T}}$-engaged to the nsp $13_{\mathrm{T}}$-apo structures, observed both by our cryo-EM (Fig. 3a) and $\mathrm{MD}$ (Fig. $3 \mathrm{c}, \mathrm{d}$ ) analyses, corresponds to a roughly $21^{\circ}$ rotation of the RecA2 domain with respect to RecA1, opening the gap between the two domains (Fig. 3a and Supplementary Video 1). The nsp $13_{\mathrm{T}}$-engaged structure is engaged with the substrate RNA and is trapped in an 'on-pathway' conformation of the nucleotide hydrolysis cycle by the nonhydrolyzable ATP analog ADP- $\mathrm{AlF}_{3}$. While the nsp $13_{\mathrm{T}}$-apo structure, being devoid of RNA, is not 'on-pathway' per se, the $21^{\circ}$ opening of the RecA2 domain from the nsp $13_{\mathrm{T}}$-engaged to $\mathrm{nsp} 13_{\mathrm{T}}$-apo conformations matches the disposition of the RecA2 domains in other SF1 helicases, such as human Upf1, a structural homolog of nsp13 (refs. ${ }^{13,24}$ ). The disposition of the RecA domains of Upf1 with $\mathrm{ADP}-\mathrm{AlF}_{3}$ and RNA substrate (Protein Data Bank (PDB) 2XZO) ${ }^{34}$ matches the nsp13 ${ }_{\mathrm{T}}$-engaged structure. On the other hand, the RecA domains in a structure of Upf1 with ADP (so likely on-pathway) are opened by a $24^{\circ}$ rotation about the same axis as the $21^{\circ}$ opening of the nsp $13_{\mathrm{T}}$-apo RecA domains (PDB 2GK6) ${ }^{35}$. We thus infer that the nsp $13_{\mathrm{T}}$-apo conformation reports on an on-pathway conformation of the RecA domains, such as in the ADP- $\mathrm{Mg}^{2+} / \mathrm{RNA}$-bound state of the translocation cycle (Fig. 3c,d). Due to the opening of the nsp13 ${ }_{\mathrm{T}}$ RecA domains, the center-of-gravity of RecA2 shifts roughly parallel with the RNA backbone by $3.4 \AA$, corresponding to the rise between stacked RNA bases. This observation is suggestive of an 'inchworm' model for translocation (Supplementary Video 1), as proposed for related SF1 helicase translocation on single-stranded nucleic acids $^{14,17,36-40}$.

Previous structural analysis of the nsp $13_{2}$-RTC identified that the $n s p 13_{\mathrm{T}}$ helicase and the RdRp translocate on the t-RNA with opposing polarities ${ }^{20}$. In circumstances where RdRp elongation of the p-RNA is hindered (such as in the event of a misincorporation at the p-RNA $3^{\prime}$ end), nsp $13_{\mathrm{T}}$ translocation activity could backtrack the RdRp ${ }^{20}$, as shown by follow-up structural and biochemical analyses ${ }^{27}$. The opposing polarities of the RdRp and nsp13 translocation activities (Extended Data Fig. 1) presented a conundrum that was not addressed by these previous studies; how is it possible for the RdRp to rapidly and efficiently synthesize RNA if it is constantly being opposed by nsp13? Moreover, the predominant complex present in the nsp13-RTC samples is the nsp $13_{2}-\mathrm{RTC}$ complex (Supplementary Table 1 ), but only nsp $13_{\mathrm{T}}$ was seen to engage with the $t-R N A$; what is the role of nsp $13_{\mathrm{P}}$ the second copy of nsp13 in the nsp $13_{2}$-RTC? The work herein suggests answers to both questions.

Maximum likelihood classification approaches revealed four distinct conformations of the nsp13 protomers in the nsp13 - RTC (Figs. 1 and 6 and Supplementary Videos 1 and 2 ). Thensp13 ${ }_{\mathrm{T}}$-engaged state resolves nsp13 ${ }_{\mathrm{T}}$ clamped onto the single-stranded downstream t-RNA, providing an atomic view of nsp13-engaged with the 


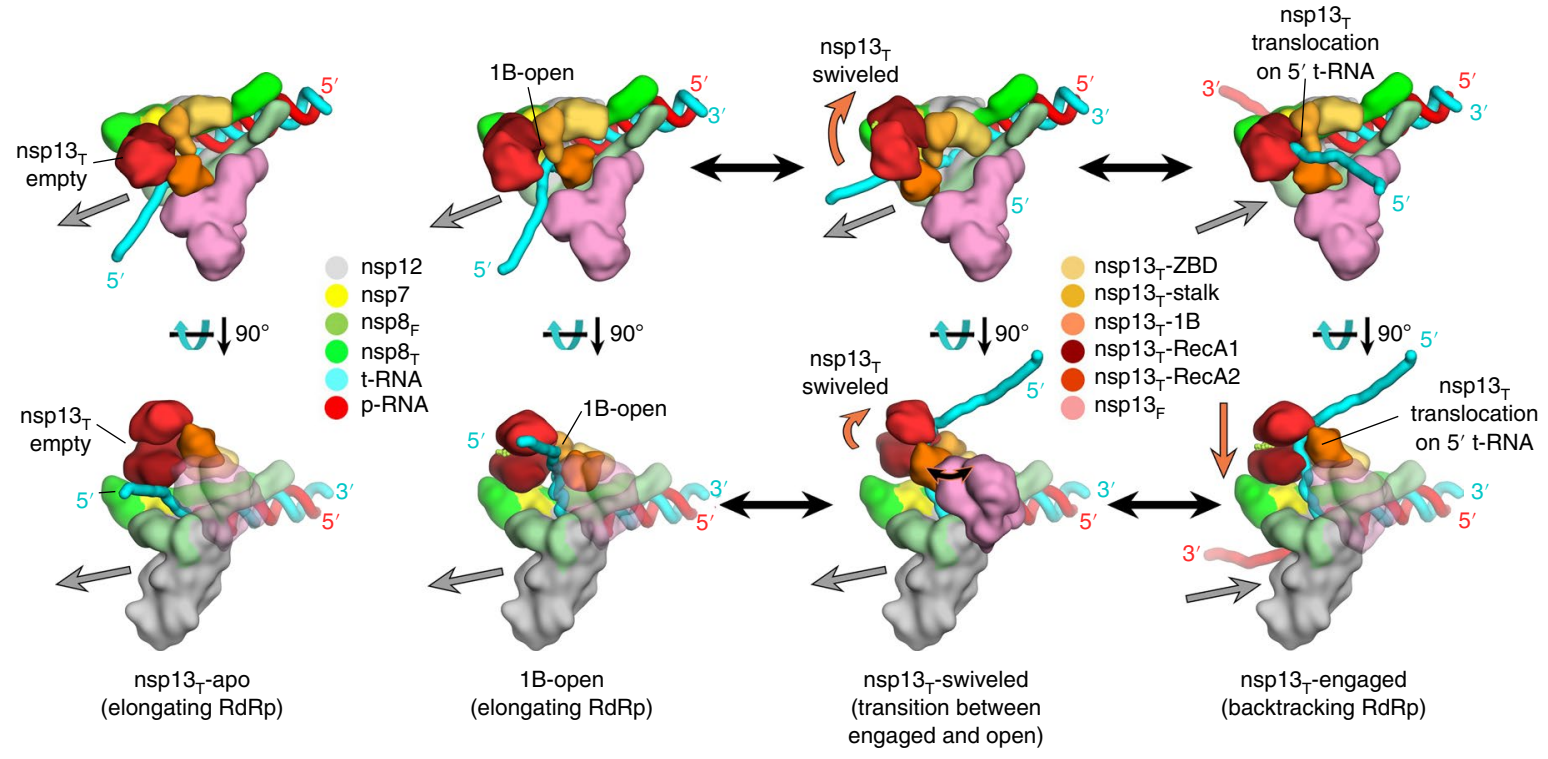

Fig. 6 | Schematic model for RTC elongation (1B-open) versus backtracking (nsp13 ${ }_{\mathrm{T}}$-engaged) states. Top views (top row) and side views (bottom row) of each structural class. Nsp13 $3_{T}$-apo (17\% of the particles): the nsp13 ${ }_{T}$ RecA domains are open, consistent with the absence of nucleotide. $\mathrm{Nsp}_{\mathrm{T}}$ is therefore not engaged with the downstream 5' t-RNA and the RdRp can freely translocate on the t-RNA with concurrent elongation of the p-RNA (gray arrow pointing downstream). 1B-open (33\%): the nsp13 $1 \mathrm{~T}$ domain is rotated open and sterically trapped by the presence of nsp13 $\mathrm{F}_{\mathrm{F}}$. The $n s p 13_{T}$ is therefore unable to engage with the downstream $5^{\prime} t-R N A$ and is inactive. The RdRp is able to elongate freely in the downstream direction. Nsp13 ${ }_{T}$-swiveled (17\%): the rotation of the $n s p 13_{T}$ protomer away from nsp13 $3_{F}$ provides space for the $n s p 13_{T} 1 B$ domain to open and/or close. We therefore propose that nsp13 $\mathrm{T}_{\mathrm{T}}$-swiveled represents a transition state between the 1B-open (elongating) and nsp13 $\mathrm{T}_{\mathrm{T}}$-engaged (backtracking) states. Nsp13 $\mathrm{T}_{\mathrm{T}}$-engaged (33\%): the nsp13 1 1 and RecA domains are clamped onto the downstream 5' t-RNA. In this state, nsp13 ${ }_{T}$ can translocate on the t-RNA in the $5^{\prime} \rightarrow 3^{\prime}$ direction (shown by the orange arrow). This counteracts RdRp elongation and causes backtracking (backward motion of the RdRp on the RNA, shown by the gray arrow pointing upstream) (Extended Data Fig. 8 and Supplementary Videos 1 and 2).

single-stranded RNA (Fig. 2). The single-stranded t-RNA threads through a tunnel formed by a deep groove between the RecAl and RecA2 domains and further enclosed by the 1B domain (Fig. 4a).

By contrast, the $1 \mathrm{~B}$-open state shows nsp $13_{\mathrm{T}}$ adopting a conformation in which the $1 \mathrm{~B}$ domain is rotated open roughly $85^{\circ}$ about the stalk domain, leaving an open RNA-binding groove (Fig. 4b). In this state, the single-stranded downstream t-RNA does not engage with the helicase. Thus, this represents an inactive state of the helicase that would be unable to translocate on the RNA.

Our structural analysis combined with MD simulations confirmed that the conformation of the $n s p 13_{\mathrm{T}} 1 \mathrm{~B}$ domain in the $1 \mathrm{~B}$-open structure is not stable on its own but is sterically trapped by the presence of nsp $13_{\mathrm{F}}$ which blocks the conformational change required for $1 \mathrm{~B}$ domain closure (Fig. $5 \mathrm{a}$ ). These results indicate that the $1 \mathrm{~B}$-open state represents a rapidly elongating state of the nsp $13_{2}-$ RTC, where the downstream single-stranded t-RNA feeds into the RdRp active site without engaging with nsp $13_{\mathrm{T}}$. Nsp13 $13_{\mathrm{F}}$ may trap the 1B-open (inactive) state of $n s p 13_{\mathrm{T}}$, allowing RdRp elongation to proceed without opposition from the nsp $13_{\mathrm{T}}$ helicase (Fig. 6). Finally, swiveling of $n s p 13_{\mathrm{T}}$ in the swiveled state allows space for the 1B-open to 1B-closed transition (Fig. $5 \mathrm{c}$ ), suggesting that the swiveled state represents a transition state between the open and closed states of the $1 \mathrm{~B}$ domain (Fig. 6). We note that the presence of nsp $13_{\mathrm{F}}$ in the nsp $13_{\mathrm{T}}$-engaged state would also block the $1 \mathrm{~B}$-closed to $1 \mathrm{~B}$-open transition, suggesting how $\mathrm{nsp} 13_{\mathrm{F}}$ can enhance the helicase activity of $n s p 13_{\mathrm{T}}$ (ref. ${ }^{21}$ ).

Thus, our results indicate a mechanism for the nsp $13_{2}-\mathrm{RTC}$ to turn backtracking on and off: switching between rapid RNA synthesis (1B-open state, elongating RdRp, Fig. 6) and backtracking (nsp13 ${ }_{\mathrm{T}}$-engaged, backtracking RdRp, Fig. 6). In our analysis of the conformational states of the nsp13 $3_{2}$ RTC, the particles were

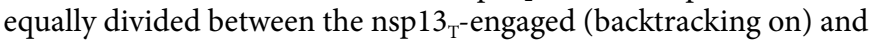

1B-open (backtracking off) states (Extended Data Figs. 3 and 8). An identical analysis of the backtracked nsp13 3 -BTC (ref. ${ }^{27}$ ) revealed a different distribution of particles in which the nsp $13_{\mathrm{T}}$-engaged (backtracking on) state was heavily favored (Extended Data Fig. 8). This raises the possibility that the conformational switch that turns backtracking on and off is allosterically controlled.

In MD simulations exploring the dynamics of the p-RNA $3^{\prime}$ nucleotide of a pretranslocated RTC, a mismatched p-RNA $3^{\prime}$ nucleotide frayed from the t-RNA toward and into the NTP-entry tunnel (which also serves as the backtracking tunnel, Supplementary Video 1 ), while a p-RNA $3^{\prime}$ nucleotide engaged in a Watson-Crick base pair with the t-RNA did not ${ }^{27}$. We thus suggest that misincorporation by the RdRp leads to fraying of the p-RNA 3' nucleotide into the NTP-entry tunnel, which may allosterically signal the rapidly elongating $1 \mathrm{~B}$-open state to switch to the backtracking $n s p 13_{\mathrm{T}}$-engaged state (via the nsp $13_{\mathrm{T}}$-swiveled state, Fig. 6). This facilitates a possible proofreading mechanism since backtracking would extrude the mismatched p-RNA $3^{\prime}$ nucleotide out of the NTP-entry tunnel (Fig. 6) ${ }^{27}$, allowing the nsp10/nsp14 $3^{\prime}$ exonuclease proofreading activity to access and degrade the mismatched p-RNA $3^{\prime}$ nucleotide $^{41-43}$. The nsp14-mediated proofreading activity is crucial for the virus to avoid mutation catastrophe while replicating its roughly $30-\mathrm{kb}$ genome $\mathrm{e}^{42}$, and is also an important determinant of SARS-CoV-2 susceptibility to many antiviral nucleotide analogs ${ }^{44}$.

\section{Online content}

Any methods, additional references, Nature Research reporting summaries, source data, extended data, supplementary information, acknowledgements, peer review information; details of author contributions and competing interests; and statements of data and code availability are available at https://doi.org/10.1038/ s41594-022-00734-6. 
Received: 5 September 2021; Accepted: 31 January 2022;

Published online: 8 March 2022

\section{References}

1. Wu, F. et al. A new coronavirus associated with human respiratory disease in China. Nature 579, 265-269 (2020).

2. Zhou, P. et al. A pneumonia outbreak associated with a new coronavirus of probable bat origin. Nature 579, 270-273 (2020).

3. Subissi, L. et al. One severe acute respiratory syndrome coronavirus protein complex integrates processive RNA polymerase and exonuclease activities. Proc. Natl Acad. Sci. USA 111, E3900-E3909 (2014).

4. Kirchdoerfer, R. N. \& Ward, A. B. Structure of the SARS-CoV nsp12 polymerase bound to nsp7 and nsp8 co-factors. Nat. Commun. 10, 2342-2349 (2019).

5. Hillen, H. S. et al. Structure of replicating SARS-CoV-2 polymerase. Nature https://doi.org/10.1038/s41586-020-2368-8 (2020).

6. Gordon, C. J. et al. Remdesivir is a direct-acting antiviral that inhibits RNA-dependent RNA polymerase from severe acute respiratory syndrome coronavirus 2 with high potency. J. Biol. Chem. 295, 6785-6797 (2020).

7. Tchesnokov, E. P. et al. Template-dependent inhibition of coronavirus RNA-dependent RNA polymerase by remdesivir reveals a second mechanism of action. J. Biol. Chem. 295, 16156-16165 (2020).

8. Kokic, G. et al. Mechanism of SARS-CoV-2 polymerase stalling by remdesivir. Nat. Commun. 12, 279 (2021).

9. Snijder, E. J., Decroly, E. \& Ziebuhr, J. The nonstructural proteins directing coronavirus RNA synthesis and processing. Adv. Virus Res. 96, 59-126 (2016)

10. van Dinten, L. C., van Tol, H., Gorbalenya, A. E. \& Snijder, E. J. The predicted metal-binding region of the arterivirus helicase protein is involved in subgenomic mRNA synthesis, genome replication, and virion biogenesis. J. Virol. 74, 5213-5223 (2000).

11. Seybert, A., Dinten, L. C., van, Snijder, E. J. \& Ziebuhr, J. Biochemical characterization of the equine arteritis virus helicase suggests a close functional relationship between arterivirus and coronavirus helicases. J. Virol. 74, 9586-9593 (2000).

12. Seybert, A. et al. A complex zinc finger controls the enzymatic activities of nidovirus helicases. J. Virol. 79, 696-704 (2005).

13. Lehmann, K. C., Snijder, E. J., Posthuma, C. C. \& Gorbalenya, A. E. What we know but do not understand about nidovirus helicases. Virus Res. 202, 12-32 (2015).

14. Saikrishnan, K., Powell, B., Cook, N. J., Webb, M. R. \& Wigley, D. B. Mechanistic basis of $5^{\prime}-3^{\prime}$ translocation in SF1B helicases. Cell 137, 849-859 (2009).

15. Adedeji, A. O. et al. Mechanism of nucleic acid unwinding by SARS-CoV helicase. PLoS ONE 7, e36521 (2012).

16. Mickolajczyk, K. J. et al. Force-dependent stimulation of RNA unwinding by SARS-CoV-2 nsp13 helicase. Biophys. J. https://doi.org/10.1016/j. bpj.2020.11.2276 (2020).

17. Singleton, M. R., Dillingham, M. S. \& Wigley, D. B. Structure and mechanism of helicases and nucleic acid translocases. Annu. Rev. Biochem. 76, 23-50 (2007)

18. Hao, W. et al. Crystal structure of Middle East respiratory syndrome coronavirus helicase. PLoS Pathog. 13, e1006474 (2017)

19. Jia, Z. et al. Delicate structural coordination of the Severe Acute Respiratory Syndrome coronavirus Nsp13 upon ATP hydrolysis. Nucleic Acids Res. 47, 6538-6550 (2019)

20. Chen, J. et al. Structural basis for helicase-polymerase coupling in the SARS-CoV-2 replication-transcription complex. Cell 182, 1560-1573.e13 (2020)

21. Yan, L. et al. Architecture of a SARS-CoV-2 mini replication and transcription complex. Nat. Commun. 11, 5874 (2020).

22. Yan, L. et al. Cryo-EM structure of an extended SARS-CoV-2 replication and transcription complex reveals an intermediate state in cap synthesis. Cell $\mathbf{1 8 4}$ 184-193.e10 (2021).
23. Hillen, H. S. Structure and function of SARS-CoV-2 polymerase. Curr. Opin. Virol. 48, 82-90 (2021).

24. Deng, Z. et al. Structural basis for the regulatory function of a complex zinc-binding domain in a replicative arterivirus helicase resembling a nonsensemediated mRNA decay helicase. Nucleic Acids Res. 42, 3464-3477 (2013).

25. Tavares, R. et al. The global and local distribution of RNA structure throughout the SARS-CoV-2 Genome. J. Virol. https://doi.org/10.1128/ JVI.02190-20 (2021)

26. Huston, N. C. et al. Comprehensive in vivo secondary structure of the SARS-CoV-2 genome reveals novel regulatory motifs and mechanisms. Mol. Cell 81, 584-598.e5 (2021).

27. Malone, B. et al. Structural basis for backtracking by the SARS-CoV-2 replication-transcription complex. Proc. Natl Acad. Sci. USA 118, e2102516118 (2021)

28. Seifert, M. et al. Inhibition of SARS-CoV-2 polymerase by nucleotide analogs from a single-molecule perspective. eLife e70968 (2021).

29. Scheres, S. H. W. RELION: implementation of a Bayesian approach to cryo-EM structure determination. J. Struct. Biol. 180, 519-530 (2012).

30. Cardone, G., Heymann, J. B. \& Steven, A. C. One number does not fit all: mapping local variations in resolution in cryo-EM reconstructions. J. Struct. Biol. 184, 226-236 (2013).

31. Bai, X., Rajendra, E., Yang, G., Shi, Y. \& Scheres, S. H. W. Sampling the conformational space of the catalytic subunit of human $\gamma$-secretase. eLife 4, e11182 (2015).

32. Yan, L. et al. Coupling of N7-methyltransferase and $3^{\prime}-5^{\prime}$ exoribonuclease with SARS-CoV-2 polymerase reveals mechanisms for capping and proofreading. Cell 184, 3474-3485.e11 (2021).

33. Newman, J. A. et al. Structure, Mechanism and crystallographic fragment screening of the SARS-CoV-2 NSP13 helicase. Nat. Commun. 12, 4848-4858 (2021).

34. Chakrabarti, S. et al. Molecular mechanisms for the RNA-dependent ATPase activity of Upf1 and its regulation by Upf2. Mol. Cell 41, 693-703 (2011).

35. Cheng, Z., Muhlrad, D., Lim, M. K., Parker, R. \& Song, H. Structural and functional insights into the human Upf1 helicase core. EMBO J. 26, 253-264 (2007).

36. Dillingham, M. S., Wigley, D. B. \& Webb, M. R. Demonstration of unidirectional single-stranded DNA translocation by PcrA helicase: measurement of step size and translocation speed. Biochem. 39, 205-212 (2000).

37. Lohman, T. M., Tomko, E. J. \& Wu, C. G. Non-hexameric DNA helicases and translocases: mechanisms and regulation. Nat. Rev. Mol. Cell Bio. 9 391-401 (2008)

38. Tomko, E. J., Fischer, C. J., Niedziela-Majka, A. \& Lohman, T. M. A nonuniform stepping mechanism for $E$. coli UvrD monomer translocation along single-stranded DNA. Mol. Cell 26, 335-347 (2007).

39. Velankar, S. S., Soultanas, P., Dillingham, M. S., Subramanya, H. S. \& Wigley, D. B. Crystal structures of complexes of PcrA DNA helicase with a DNA substrate indicate an inchworm mechanism. Cell 97, 75-84 (1999).

40. Yarranton, G. T. \& Gefter, M. L. Enzyme-catalyzed DNA unwinding: etudies on Escherichia coli rep protein. Proc. Natl Acad. Sci. USA 76, 1658-1662 (1979).

41. Minskaia, E. et al. Discovery of an RNA virus $3^{\prime} \rightarrow 5^{\prime}$ exoribonuclease that is critically involved in coronavirus RNA synthesis. Proc. Natl Acad. Sci. USA 103, 5108-5113 (2006).

42. Gorbalenya, A. E., Enjuanes, L., Ziebuhr, J. \& Snijder, E. J. Nidovirales: evolving the largest RNA virus genome. Virus Res. 117, 17-37 (2006)

43. Denison, M. R., Graham, R. L., Donaldson, E. F., Eckerle, L. D. \& Baric, R. S. Coronaviruses: an RNA proofreading machine regulates replication fidelity and diversity. RNA Biol. 8, 270-279 (2011).

44. Agostini, M. L. et al. Coronavirus susceptibility to the antiviral remdesivir (GS-5734) is mediated by the viral polymerase and the proofreading exoribonuclease. MBio. 9, e00221-18 (2018).

Publisher's note Springer Nature remains neutral with regard to jurisdictional claims in published maps and institutional affiliations.

(c) The Author(s), under exclusive licence to Springer Nature America, Inc. 2022 


\section{Methods}

No statistical methods were used to predetermine sample size. The experiments were not randomized, and the investigators were not blinded to allocation during experiments and outcome assessment.

Protein expression and purification. SARS-CoV-2 nsp 12 was expressed and purified as described in ref. ${ }^{20}$. Briefly, a pRSFDuet-1 plasmid containing $\mathrm{His}_{6}$-small ubiquitin-like modifier (SUMO) SARS-CoV-2 nsp12 (Addgene no. 159107) was transformed into Escherichia coli BL21-CodonPlus cells (Agilent). Cells were grown and protein expression was induced by the addition of isopropyl $\beta$-D1-thiogalactopyranoside (IPTG). Cells were collected and lysed in a French press (Avestin). The lysate was cleared by centrifugation and purified on a HiTrap Heparin HP column (Cytiva). The fractions containing nsp12 were loaded onto a HisTrap HP column (Cytiva) for further purification. Eluted nsp12 was dialyzed, cleaved with $\mathrm{His}_{6}$-Ulp1 SUMO protease and passed through a HisTrap HP column to remove the SUMO protease. Flow-through was collected, concentrated by centrifugal filtration (Amicon), and loaded on a Superdex 200 Hiload 16/600 (Cytiva). Glycerol was added to the purified nsp12, aliquoted, flash-frozen with liquid $\mathrm{N}_{2}$ and stored at $-80^{\circ} \mathrm{C}$.

SARS-CoV-2 nsp7/8 was expressed and purified as described in ref. ${ }^{20}$. Briefly, the pCDFDuet-1 plasmid containing $\mathrm{His}_{6}$ SARS-CoV-2 nsp7/8 (Addgene no. 159092) was transformed into E. coli BL21 (DE3). Cells were grown and protein expression was induced by the addition of IPTG. Cells were collected and lysed in a French press (Avestin). The lysate was cleared by centrifugation and purified on a HisTrap HP column (Cytiva). Eluted nsp7/8 was dialyzed, cleaved with $\mathrm{His}_{6}$-Prescission Protease to cleave $\mathrm{His}_{6}$ tag and then passed through a HisTrap HP column to remove the protease (Cytiva). Flow-through was collected, concentrated by centrifugal filtration (Amicon) and loaded onto a Superdex 75 Hiload 16/600 (Cytiva). Glycerol was added to the purified nsp7/8, aliquoted, flash-frozen with liquid $\mathrm{N}_{2}$ and stored at $-80^{\circ} \mathrm{C}$.

SARS-CoV-2 nsp13 was expressed and purified as described in ref. ${ }^{20}$. Briefly, the pet 28 plasmid containing $\mathrm{His}_{6}$ SARS-CoV-2 nsp13 (Addgene no. 159390) was transformed into E. coli Rosetta (DE3) (Novagen). Cells were grown and protein expression was induced by the addition of IPTG. Cells were collected and lysed in a French press (Avestin). The lysate was cleared by centrifugation and purified on a HisTrap HP column (Cytiva). Eluted nsp13 was dialyzed, cleaved with $\mathrm{His}_{6}$-Prescission Protease and then passed through a HisTrap HP column to remove protease (Cytiva). Flow-through was collected, concentrated by centrifugal filtration (Amicon) and loaded onto a Superdex 200 Hiload 16/600 (Cytiva). Glycerol was added to the purified nsp13, aliquoted, flash-frozen with liquid $\mathrm{N}_{2}$ and stored at $-80^{\circ} \mathrm{C}$.

Preparation of SARS-CoV-2 nsp13-RTC for cryo-EM. Cryo-EM samples of SARS-CoV-2 nsp13-RTC were prepared as described in ref. ${ }^{20}$. Briefly, purified nsp12 and nsp7/8 were concentrated, mixed in a 1:3 molar ratio and incubated for $20 \mathrm{~min}$ at $22^{\circ} \mathrm{C}$. Annealed RNA scaffold (Horizon Discovery, Ltd) was added to the nsp7/8/12 mixture and incubated for $15 \mathrm{~min}$ at $22^{\circ} \mathrm{C}$. Sample was buffer exchanged into cryo-EM buffer (20 mM HEPES pH 8.0, $150 \mathrm{mM} \mathrm{K}$-Acetate, $10 \mathrm{mM} \mathrm{MgCl}_{2}$, $2 \mathrm{mM} \mathrm{DTT}$ ) and further incubated for $20 \mathrm{~min}$ at $30^{\circ} \mathrm{C}$. The sample was purified over a Superose 6 Increase 10/300 GL column (Cyriva) in cryo-EM buffer. The peak corresponding to nsp7/8/12/RNA complex was pooled and concentrated by centrifugal filtration (Amicon). Purified nsp13 was concentrated by centrifugal filtration (Amicon) and buffer exchanged into cryo-EM buffer. Buffer exchanged nsp13 was mixed with ADP ( $1 \mathrm{mM}$ final $)$ and $\mathrm{AlF}_{3}(1 \mathrm{mM}$ final $)$ and then added to nsp7/8/12/RNA at a molar ratio of $1: 1$. Complex was then incubated for $5 \mathrm{~min}$ at $30^{\circ} \mathrm{C}$.

Cryo-EM grid preparation. Before grid freezing, 3-(3-cholamidopropyldimethylammonio)-2-hydroxy-1-propanesulfonate (CHAPSO, Anatrace) was added to the sample ( $8 \mathrm{mM}$ final), resulting in a final complex concentration of $8 \mu \mathrm{M}$. The final buffer condition for the cryo-EM sample was $20 \mathrm{mM}$ HEPES $\mathrm{pH}$ 8.0, $150 \mathrm{mM}$ K-acetate, $10 \mathrm{mM} \mathrm{MgCl}$, $2 \mathrm{mM}$ DTT, $1 \mathrm{mM}$ ADP, $1 \mathrm{mM}$ $\mathrm{AlF}_{3}, 8 \mathrm{mM}$ CHAPSO. C-flat holey carbon grids (CF-1.2/1.3-4Au, EMS) were glow-discharged for $20 \mathrm{~s}$ before the application of $3.5 \mu \mathrm{l}$ of sample. Using a Vitrobot Mark IV (Thermo Fisher Scientific), grids were blotted and plunge-frozen into liquid ethane with $90 \%$ chamber humidity at $4^{\circ} \mathrm{C}$.

Cryo-EM data acquisition and processing. Structural biology software was accessed through the SBGrid consortium ${ }^{45}$. Grids were imaged using a $300 \mathrm{kV}$ Titan Krios (Thermo Fisher Scientific) equipped with a GIF BioQuantum and K3 camera (Gatan). Images were recorded with Leginon ${ }^{46}$ with a pixel size of $1.07 \AA$ per pixel (px) (micrograph dimensions of $5,760 \times 4,092 \mathrm{px}$ ) over a defocus range of -0.8 to $-2.5 \mu \mathrm{m}$ with a $20-\mathrm{eV}$ slit. Videos were recorded in 'counting mode' (native K3 camera binning 2) with roughly $30 \mathrm{e}^{-}$per px per $\mathrm{s}$ in dosefractionation mode with subframes of $50 \mathrm{~ms}$ over a $2.5 \mathrm{~s}$ exposure (50 frames) to give a total dose of roughly $66 \mathrm{e}^{-} / \AA^{2}$. Dose-fractionated videos were gain-normalized, drift-corrected, summed and dose-weighted using MotionCor2 (ref. ${ }^{47}$ ). The contrast transfer function (CTF) was estimated for each summed image using the Patch CTF module in cryoSPARC v.2.15.0 (ref. ${ }^{48}$ ). Particles were picked and extracted from the dose-weighted images with box size of $256 \mathrm{px}$ using cryoSPARC Blob Picker and Particle Extraction. The entire dataset consisted of 17,806 motion-corrected images with 3,750,107 particles. Particles were sorted using two rounds of cryoSPARC two-dimensional classification $(N=100$, where $N$ equals the number of classes), resulting in 661,105 curated particles that were re-extracted with a boxsize of $320 \mathrm{px}$. An initial model was generated using cryoSPARC ab initio reconstruction $(N=3)$ on a subset of the particles. Particles were further curated using this initial model as a three-dimensional (3D) template for cryoSPARC Heterogeneous Refinement $(N=3)$, resulting in 451,760 particles (green map, Extended Data Figs. 2 and 3). Curated particles were further classified using cryoSPARC Heterogeneous Refinement $(N=3)$. Each of the resulting 3D classes were further processed with cryoSPARC Ab Initio Reconstruction $(N=3)$, generating three distinct models that could be used to sort particles (ref. nsp13 1 -RTC; ref. ${ }^{2}$ nsp $13_{2}-$ RTC; ref. $^{3}$ (nsp $13_{2}-$ RTC) $)_{2}$ ). Using refs. ${ }^{1-3}$ as 3D templates for heterogeneous Refinement $(N=6)$, multi-reference classification was performed on the 451,760 curated particles. Classification revealed three unique classes: nsp13 1 -RTC (map 2; 85,206 particles, yellow), nsp $13_{2}-$ RTC (map 3; 315,216 particles, red) and (nsp13 - RTC) (map 4; 35,403 particles, blue). Particles within each class were further processed using RELION 3.1-beta Bayesian Polishing ${ }^{49}$. Polished particles were refined using cryoSAPRC Local and Global CTF Refinement in combination with cryoSPARC Nonuniform Refinement ${ }^{50}$, resulting in structures with the following particle counts and nominal resolutions: nsp13 1 -RTC (85,187 particles; $3.2 \AA)$, nsp13 ${ }_{2}$-RTC (315,120 particles; $\left.2.9 \AA\right)$ and $\left(\text { nsp } 13_{2}-\mathrm{RTC}\right)_{2}(35,392$ particles; $3.3 \AA)$. To facilitate model building of nsp $13_{2}-$ RTC, particles from nsp $13_{1}-$ RTC and nsp $13_{2}-$ RTC were combined in a cryoSPARC Nonuniform Refinement, subtracted (masking the RTC), and further refined with cryoSPARC Local Refinement using a mask encompassing the RTC. The resulting map, deemed RTC (local), had nominal resolution of $2.8 \AA$. Additionally, particles from the nsp $13_{2}$-RTC were subtracted in different regions (using separate masks for nsp $12-\mathrm{NiRAN}, \mathrm{nsp} 13_{\mathrm{T}}$ and $\mathrm{nsp} 13_{\mathrm{F}}$ ) and the particles from each subtraction were further refined with masked cryoSPARC Local Refinement. The resulting maps had the following nominal resolutions: nsp13 $($ local), $3.4 \AA$; nsp13 F $_{\mathrm{F}}$ local), $3.3 \AA$ and nsp12-NiRAN(local), $2.7 \AA$. Locally refined maps were combined into an nsp $13_{2}$-RTC composite map using PHENIX 'Combine Focused Maps $^{51,52}$, with a resulting nominal resolution of $2.8 \AA$. The nsp13-RecA domains in particles from the nsp $13_{1}-\mathrm{RTC}$ and nsp $13_{2}-\mathrm{RTC}$ classes were sorted using particle subtraction (masking around the RecA domains, shown as red mesh in Extended Data Figs. 2 and 3), followed by masked RELION 3D classification. Classification of RecA domains in the nsp13 - RTC particles (pale yellow) did not reveal discrete conformational heterogeneity in the RecA domains. However, classification of RecA domains in the nsp $13_{2}$-RTC particles (light red) revealed unique conformations of the RecA domains with the following particle counts and nominal resolutions: RecA classI (52,403 particles; $3.5 \AA)$, RecA class II $(102,615$ particles; $3.1 \AA$ ) $)$, RecA class III (54,830 particles; $3.5 \AA$ A), RecA class IV $(105,272$ particles; $3.1 \AA$ ). Local-resolution calculations were generated using blocres and blocfilt from the Bsoft package ${ }^{30}$.

Model building and refinement. For an initial model of the nsp $13_{2}-\mathrm{RTC}$, the initial RTC model was derived from PDB 6XEZ (ref. ${ }^{20}$ ) and the initial nsp 13 model from PDB 6ZSL (ref. ${ }^{33}$ ). The models were manually fit into the cryo-EM density maps using Chimera ${ }^{53}$ and rigid-body and real-space refined using PHENIX real-space-refine ${ }^{51,52}$. For real-space refinement, rigid-body refinement was followed by all-atom and $B$ factor refinement with Ramachandran and secondary structure restraints. Models were inspected and modified in COOT ${ }^{54}$.

MD simulations. General simulation setup and parameterization. Proteins, RNAs and ions were parameterized with the DES-Amber SF1.0 force field ${ }^{55,56}$. ATP and ADP were parameterized using the DES-Amber nucleobase, sugar and phosphate parameters (force field files are available on request). The systems were solvated with water parameterized with the TIP4P-D water model $^{57}$ and neutralized with a $150 \mathrm{mM} \mathrm{NaCl}$ buffer. The systems of isolated nsp13 each contained roughly 160,000 atoms in a $110 \times 110 \times 110 \AA^{3}$ cubic box, and the systems of the nsp $13_{2}-\mathrm{RTC}$ complex each contained roughly 887,000 atoms in a $190 \times 190 \times 190 \AA^{3}$ cubic box.

Systems were first equilibrated on GPU Desmond using a mixed NVT/NPT schedule $^{58}$, followed by a $1-\mu$ s relaxation simulation on Anton, a special-purpose machine for MD simulations $s^{59}$. All production simulations were performed on Anton and initiated from the last frame of the relaxation simulation. Production simulations were performed in the NPT ensemble ${ }^{60}$ at $310 \mathrm{~K}$ using the MartynaTobias-Klein barostat ${ }^{61}$. The simulation time step was $2.5 \mathrm{fs}$, and a modified r-RESPA integrator ${ }^{62}$ was used in which long-range electrostatic interactions were evaluated every three time steps. Electrostatic forces were calculated using the u-series $\operatorname{method}^{63}$. A 9-Å cutoff was applied for the van der Waals calculations.

System preparation. The initial conformations of class II nsp13 $3_{\mathrm{T}}$ bound to the various substrates (ATP- $\mathrm{Mg}^{2+} / \mathrm{RNA}, \mathrm{ADP}-\mathrm{Mg}^{2+} / \mathrm{RNA}, \mathrm{ATP}-\mathrm{Mg}^{2+}$ and ADP- $\mathrm{Mg}^{2+}$ ) were prepared based on the cryo-EM structure of the class II nsp13 ${ }_{2}-\mathrm{BTC}_{5}$. The initial conformation of the class IV, 1B-open $\mathrm{nsp} 3_{\mathrm{T}}$ structure was prepared from the cryo-EM class IV nsp13 $-\mathrm{BTC}_{5}$ structure. $\mathrm{AlF}_{3}$ was removed from the active site. Missing loops and termini in proteins were capped with ACE/NME capping 
groups. In simulations with ATP at the active site, ATP was manually placed using ADP in the cryo-EM structure as the reference. The systems were prepared for simulation using the Protein Preparation Wizard in Schrödinger Maestro (Schrödinger Release 2020-4: Maestro, Schrödinger, LLC, 2020).

Simulation analysis. The average r.m.s.d. was calculated for the RecA2 domain (residues 450-690) and 1B domain (residues 145-200) of nsp13 $3_{\mathrm{T}}$ between the cryo-EM structures and instantaneous structures from the trajectories, aligned on the RecAl lobe (residues 240-440). Simulation structures shown in figures were rendered using PyMol (The PyMOL Molecular Graphics System, v.2.0 Schrödinger, LLC)

Quantification and statistical analysis. The local resolution of the cryo-EM maps (Supplementary Figs. 4 and 6) was estimated using blocres ${ }^{30}$ with the following parameters: box size 15 , sampling 1.1 and cutoff 0.5 . Directional 3DFSCs (Supplementary Figs. 4 and 6) were calculated using $3 \mathrm{DFSC}^{64}$. The quantification and statistical analyses for model refinement and validation were generated using MolProbity ${ }^{65}$ and PHENIX ${ }^{52}$.

Reporting Summary. Further information on research design is available in the Nature Research Reporting Summary linked to this article.

\section{Data availability}

All unique/stable reagents generated in this study are available without restriction from one of the lead contacts, S.A.D. (darst@rockefeller.edu). The cryo-EM density maps and atomic coordinates have been deposited in the EMDataBank and PDB as follows: nsp13 1 -RTC (EMD-24431, PDB 7RE2), nsp13 ${ }_{2}$-RTC (composite) (EMD-24430, PDB 7RE1), (nsp13 $\left.{ }_{2}-\mathrm{RTC}\right)_{2}$ (EMD-24432, PDB 7RE3), nsp13 $3_{2}$-RTC

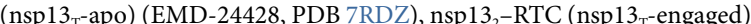
(EMD-24427, PDB 7RDY), nsp13 - RTC (nsp13 ${ }_{\mathrm{T}}$-swiveled) (EMD-24429, PDB 7RE0) and nsp13-RTC (1B-open) (EMD-24426, PDB 7RDX). The MD trajectories described in this work are available at https://www.deshawresearch. com/downloads/download_trajectory_sarscov2.cgi/.

\section{References}

45. Morin, A. et al. Collaboration gets the most out of software. eLife $\mathbf{2}$, e01456 (2013)

46. Suloway, C. et al. Automated molecular microscopy: the new Leginon system. J. Struct. Biol. 151, 41-60 (2005)

47. Zheng, S. Q. et al. MotionCor2: anisotropic correction of beam-induced motion for improved cryo-electron microscopy. Nat. Methods 14, 331-332 (2017).

48. Punjani, A., Rubinstein, J. L., Fleet, D. J. \& Brubaker, M. A. cryoSPARC: algorithms for rapid unsupervised cryo-EM structure determination. Nat. Methods 14, 290-296 (2017).

49. Zivanov, J. et al. New tools for automated high-resolution cryo-EM structure determination in RELION-3. eLife 7, e42166 (2018).

50. Punjani, A., Zhang, H. \& Fleet, D. J. Non-uniform refinement: adaptive regularization improves single-particle cryo-EM reconstruction. Nat. Methods $17,1214-1221$ (2020).

51. Afonine, P. V. et al. New tools for the analysis and validation of cryo-EM maps and atomic models. Acta Crystallogr. Sect. D., Struct. Biol. 74, 814-840 (2018).

52. Adams, P. D. et al. PHENIX: a comprehensive Python-based system for macromolecular structure solution. Acta Crystallogr., Sect. D: Biol. Crystallogr. 66, 213-221 (2010).

53. Pettersen, E. F. et al. UCSF Chimera-a visualization system for exploratory research and analysis. J. Comput. Chem. 25, 1605-1612 (2004).

54. Emsley, P. \& Cowtan, K. Coot: model-building tools for molecular graphics. Acta Crystallogr., Sect. D: Biol. Crystallogr. 60, 2126-2132 (2004).

55. Piana, S., Robustelli, P., Tan, D., Chen, S. \& Shaw, D. E. Development of a force field for the simulation of single-chain proteins and protein-protein complexes. J. Chem. Theory Comput. 16, 2494-2507 (2020).

56. Tan, D., Piana, S., Dirks, R. M. \& Shaw, D. E. RNA force field with accuracy comparable to state-of-the-art protein force fields. Proc. Natl Acad. Sci. USA 115, 201713027 (2018).

57. Piana, S., Donchev, A. G., Robustelli, P. \& Shaw, D. E. Water dispersion interactions strongly influence simulated structural properties of disordered protein states. J. Phys. Chem. B. 119, 5113-5123 (2015).
58. Bowers, K. J. et al. Scalable algorithms for molecular dynamics simulations on commodity clusters. In Proc. ACM/IEEE Conference on Supercomputing 2006, 11-17 November, Tampa, FL, USA 43-43 (IEEE, 2006).

59. Shaw, D. E. et al. Anton 2: raising the bar for performance and programmability in a special-purpose molecular dynamics supercomputer. in Proc. International Conference for High Performance Computing, Networking, Storage and Analysis, 16-21 November, New Orleans, LA, USA 41-53 (IEEE, 2014).

60. Lippert, R. A. et al. Accurate and efficient integration for molecular dynamics simulations at constant temperature and pressure. J. Chem. Phys. 139, 164106 (2013)

61. Martyna, G. J., Tobias, D. J. \& Klein, M. L. Constant pressure molecular dynamics algorithms. J. Chem. Phys. 101, 4177-4189 (1994).

62. Tuckerman, M., Berne, B. J. \& Martyna, G. J. Reversible multiple time scale molecular dynamics. J. Chem. Phys. 97, 1990-2001 (1992).

63. Predescu, C. et al. The u-series: a separable decomposition for electrostatics computation with improved accuracy. J. Chem. Phys. 152, 084113 (2020).

64. Tan, Y. Z. et al. Addressing preferred specimen orientation in single-particle cryo-EM through tilting. Nat. Methods 14, 793-796 (2017).

65. Chen, V. B. et al. MolProbity: all-atom structure validation for macromolecular crystallography. Acta Crystallogr., Sect. D: Biol. Crystallogr. 66, 12-21 (2010).

\section{Acknowledgements}

We thank M. Ebrahim and L. Urnavicius at The Rockefeller University Evelyn Gruss Lipper Cryo-electron Microscopy Resource Center and H. Kuang at the New York Structural Biology Center (NYSBC) for help with cryo-EM data collection; R. Landick, T. Appleby and members of the Darst/Campbell laboratory for helpful discussions and M. Grasso, P.M.M. Shelton, T.M. Kapoor, P.D.B. Olinares and B.T. Chait (The Rockefeller University) for helpful discussions and initial sample characterizations and analyses. Some of the work reported here was conducted at the Simons Electron Microscopy Center and the National Resource for Automated Molecular Microscopy and National Center for CryoEM Access and Training located at the NYSBC, supported by grants from the National Institutes of Health (NIH) National Institute of General Medical Sciences (no. P41 GM103310), NYSTAR, the Simons Foundation (no. SF349247), the NIH Common Fund Transformative High Resolution Cryo-Electron Microscopy program (no. U24 GM129539) and NY State Assembly Majority. This work was supported by the Pels Family Center for Biochemistry and Structural Biology (The Rockefeller University) and NIH grant nos. R01 GM114450 (E.A.C.), R35 GM118130 (S.A.D.) and R01 AI161278 (E.A.C./S.A.D.)

\section{Author contributions}

Conceptualization was developed by J.C., Q.W., B.M., J.K.P., E.A.C., D.E.S. and S.A.D. Cloning, protein purification and biochemistry were done by J.C., B.M. and E.L. Cryo-EM specimen preparation was carried out by J.C., B.M. and E.L. Cryo-EM data collection and processing were done by J.C., K.M. and E.T.E. Model building and structural analysis were carried out by J.C., B.M., E.A.C. and S.A.D. MD simulations and analysis were carried out by Q.W. and Y.P. Funding acquisition and supervision were the responsibility of E.A.C., D.E.S. and S.A.D. The first draft was written by Q.W., B.M., J.K.P., E.A.C., D.E.S. and S.A.D. All authors contributed to finalizing the manuscript.

\section{Competing interests}

The authors declare no competing interests.

\section{Additional information}

Extended data are available for this paper at https://doi.org/10.1038/ s41594-022-00734-6.

Supplementary information The online version contains supplementary material available at https://doi.org/10.1038/s41594-022-00734-6.

Correspondence and requests for materials should be addressed to David E. Shaw or Seth A. Darst.

Peer review information Nature Structural and Molecular Biology thanks Dong Wang and the other, anonymous, reviewer(s) for their contribution to the peer review of this work. Beth Moorefield was the primary editor on this article and managed its editorial process and peer review in collaboration with the rest of the editorial team. Peer reviewer reports are available.

Reprints and permissions information is available at www.nature.com/reprints. 


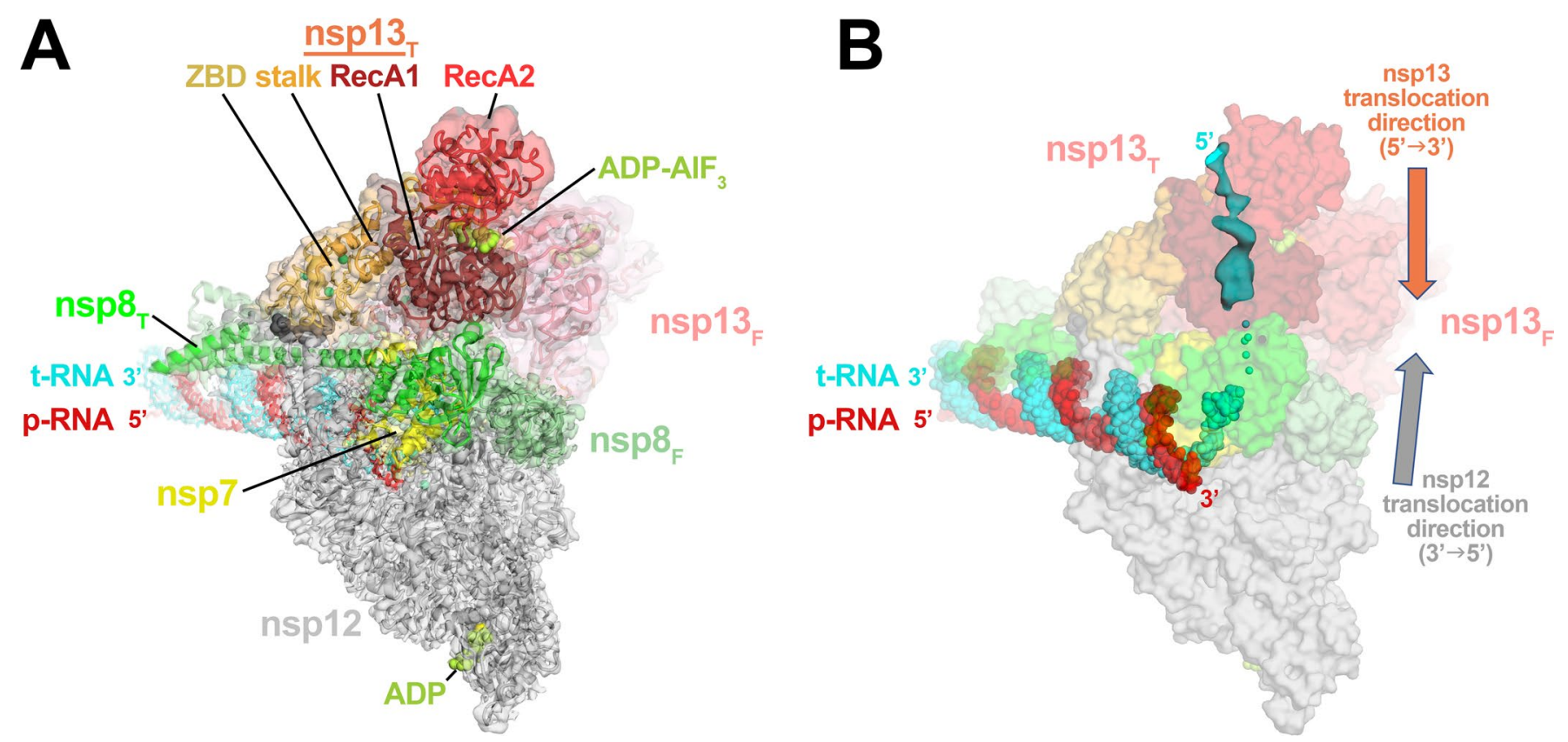

Extended Data Fig. 1 | Consensus cryo-EM structure of an nsp13 - RTC. a. Overall architecture of the consensus nsp13 ${ }_{2}-$ RTC. Shown is the transparent cryo-EM density (map3, local-resolution filtered) with the nsp13 $3_{2}-\mathrm{RTC}$ model superimposed. $\mathbf{b}$. The consensus nsp13 ${ }_{2}-\mathrm{RTC}$ structure is shown; RNA is shown as atomic spheres, proteins are shown as transparent molecular surfaces. A low-pass filtered ( $6 \AA$ ) cryo-EM difference density reveals the path of the downstream t-RNA 5'-segment through the RNA binding groove of nsp13 ${ }_{T}$ (cyan surface). 
A

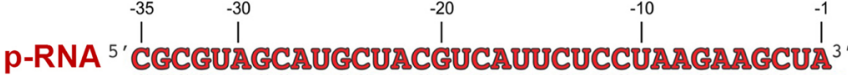

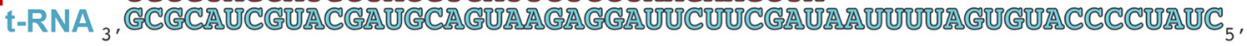

B
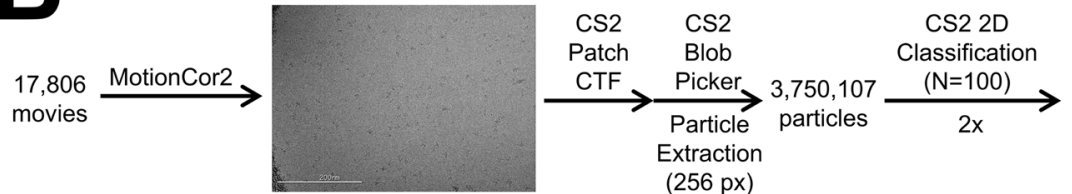

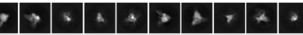

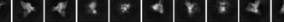

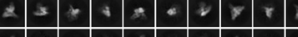

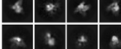

661,105 selected particles

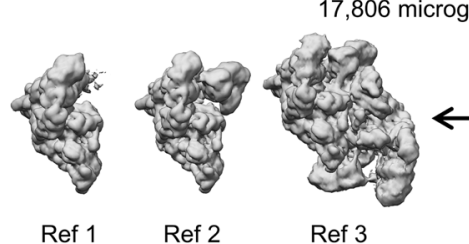

CS2

(256 px)

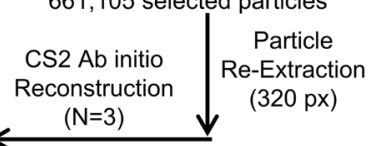

Heterogeneous Refinement $(\mathrm{N}=3)$

CS2 Ab initio

Reconstruction

$(\mathrm{N}=3$, per class $)$

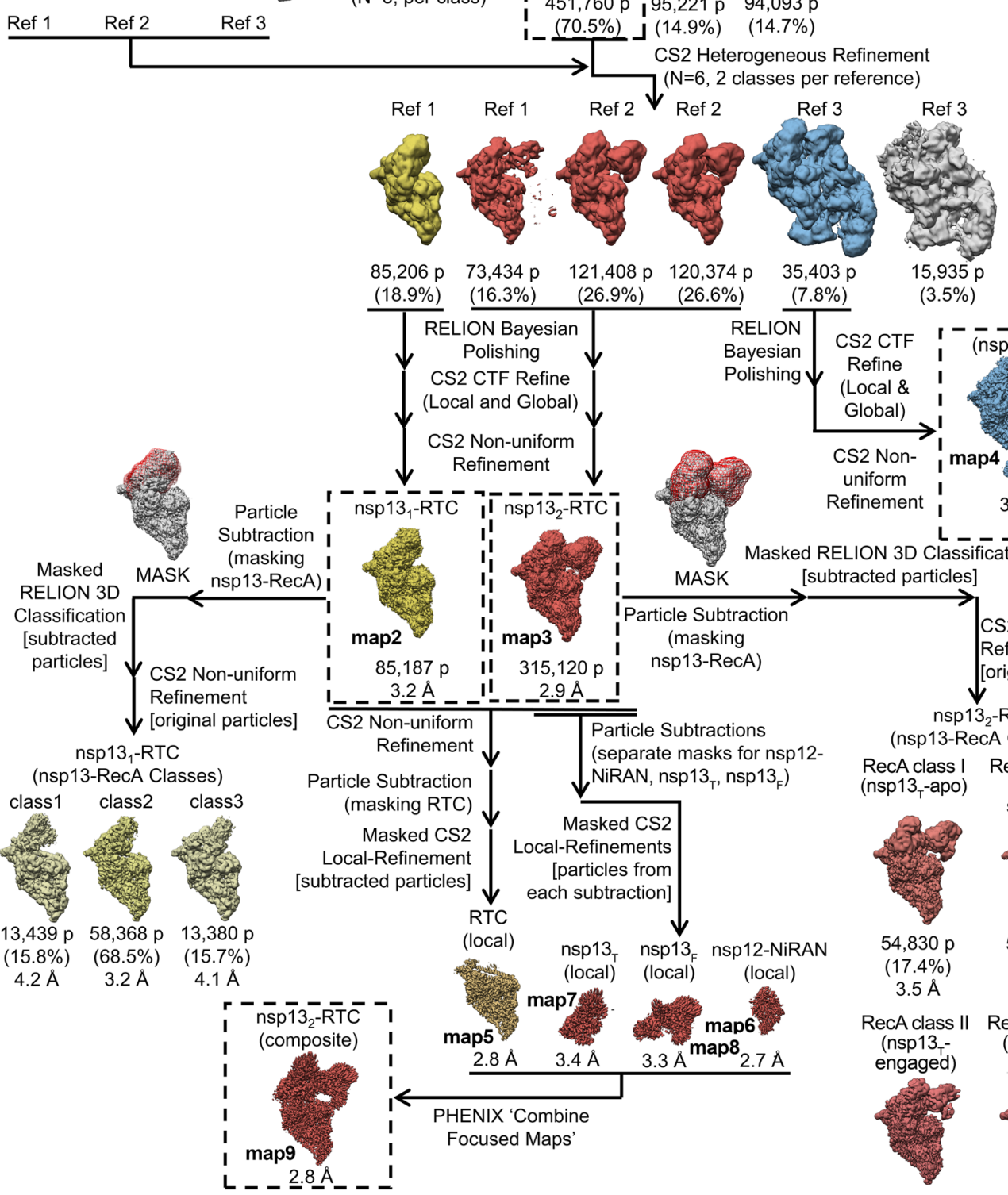

CS2 Heterogeneous
Refinement $(\mathrm{N}=3)$

map1

1 . . .

$(70.5 \%)(14.9 \%) \quad(14.7 \%)$

CS2 Heterogeneous Refinement 


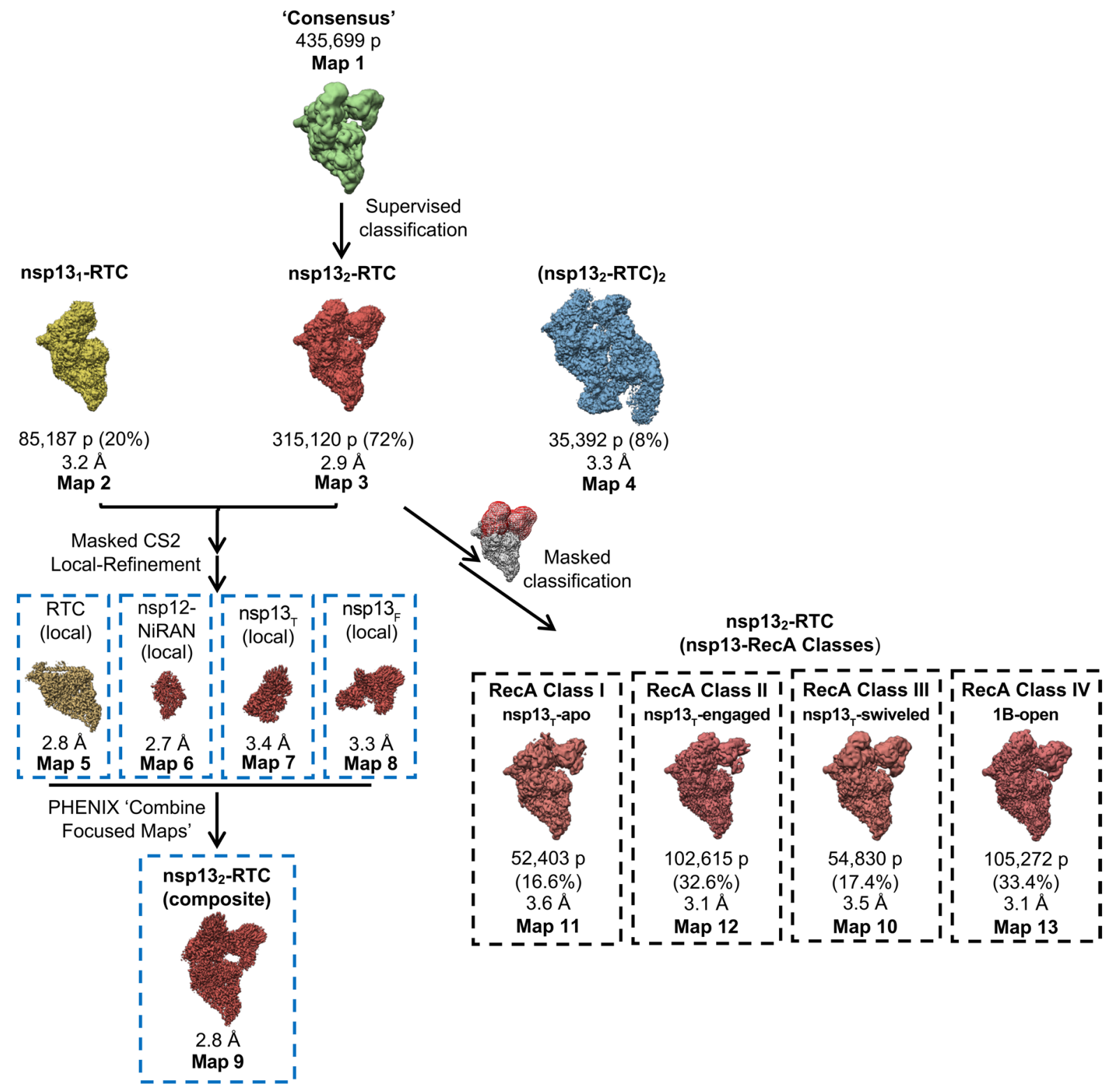

Extended Data Fig. 3 | Processing flowchart overview and map nomenclature. 


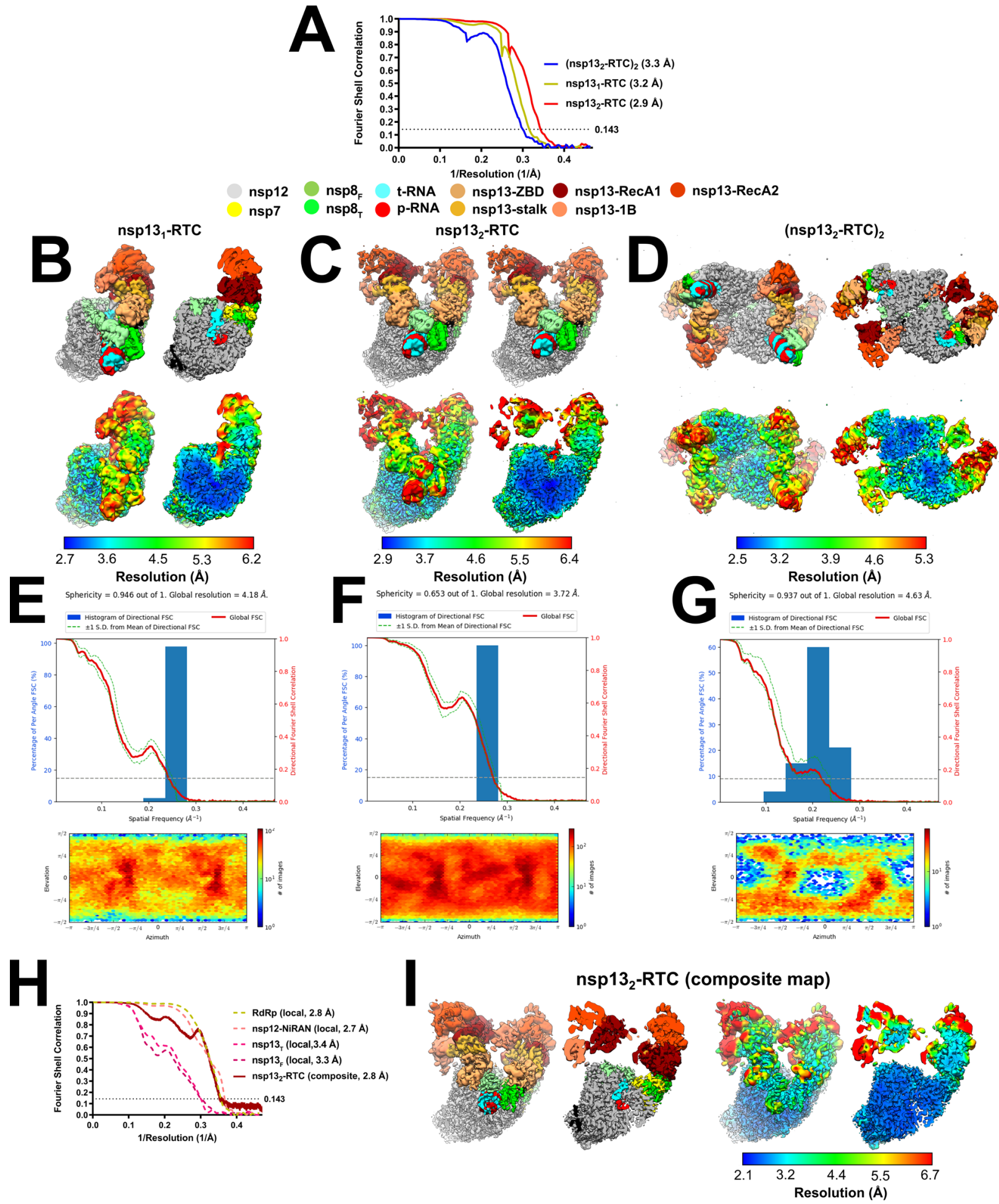

Extended Data Fig. 4 | Cryo-EM of nsp13-RTC. a. Gold-standard Fourier-shell correlation (FSC) plot for nsp13,-RTC (yellow line), nsp13 2 -RTC (red line), and (nsp13 - RTC) $)_{2}$ (blue line) calculated by comparing the independently determined half-maps from cryoSPARC. The dotted line represents the 0.143 FSC cutoff which indicates a nominal resolution of $3.2 \AA$ (nsp13 $\left.3_{2}-R T C\right), 2.9 \AA$ (nsp13 - RTC), and $3.3 \AA\left[\left(n s p 13_{2}-R T C\right)_{2}\right]$. b - d . The cryo-EM maps filtered by local resolution ${ }^{3}$ are shown. The view on the right is a cross-section. (top) Colored by subunit (key above). (bottom) Colored by local resolution (key on the bottom). b. Nsp13-RTC (map2). c. Nsp13,-RTC (map3). d. (nsp13,-RTC) (map4). e- g. Directional 3D Fourier shell correlation calculated by 3DFSC $(\text { top })^{4}$ and particle orientation distribution calculated by cryoSPARC (bottom). e. Nsp13-RTC. f. Nsp13 - RTC. g. (nsp13 2 -RTC) 2 .h. Gold-standard FSC plots for local-refined maps (dashed lines) and nsp13 - RTC composite map (map9) (solid line). i. The nsp13 - -RTC composite map (map9) filtered by local resolution ${ }^{3}$. (left) Colored by subunit. (right) Colore by local resolution (key at the bottom). 


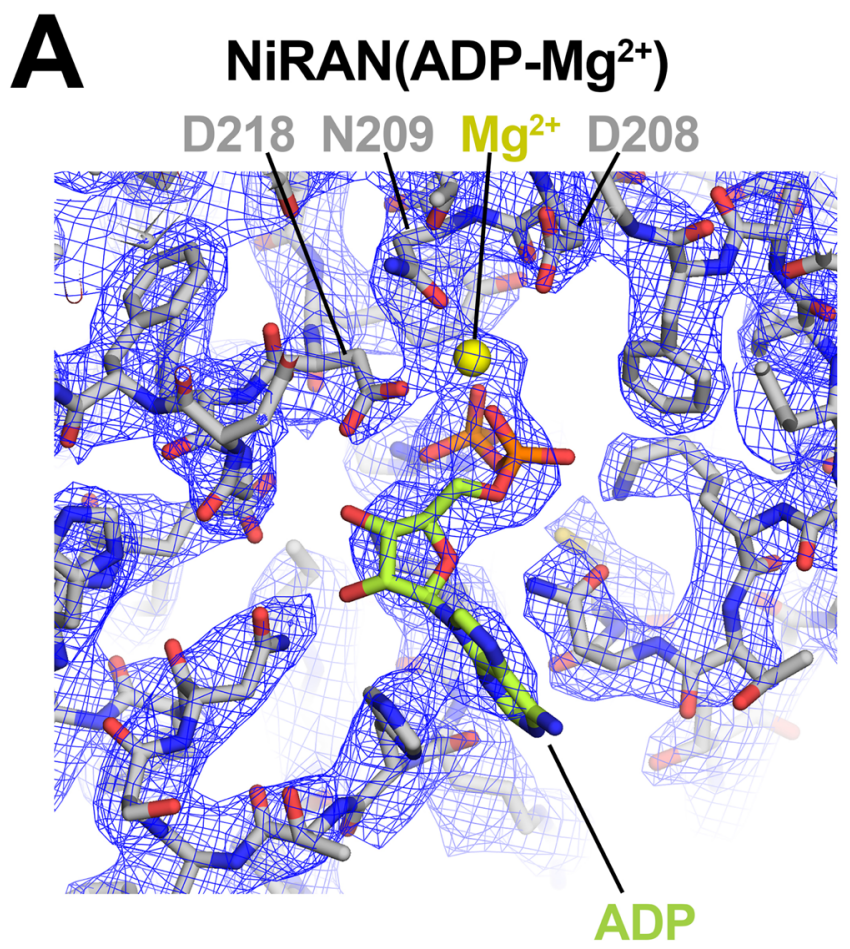

\section{D $\quad$ nsp13 ${ }_{\mathrm{T}}\left(\mathrm{ADP}_{-}-\mathrm{Mg}^{2+}-\mathrm{AlF}_{3}\right)$}

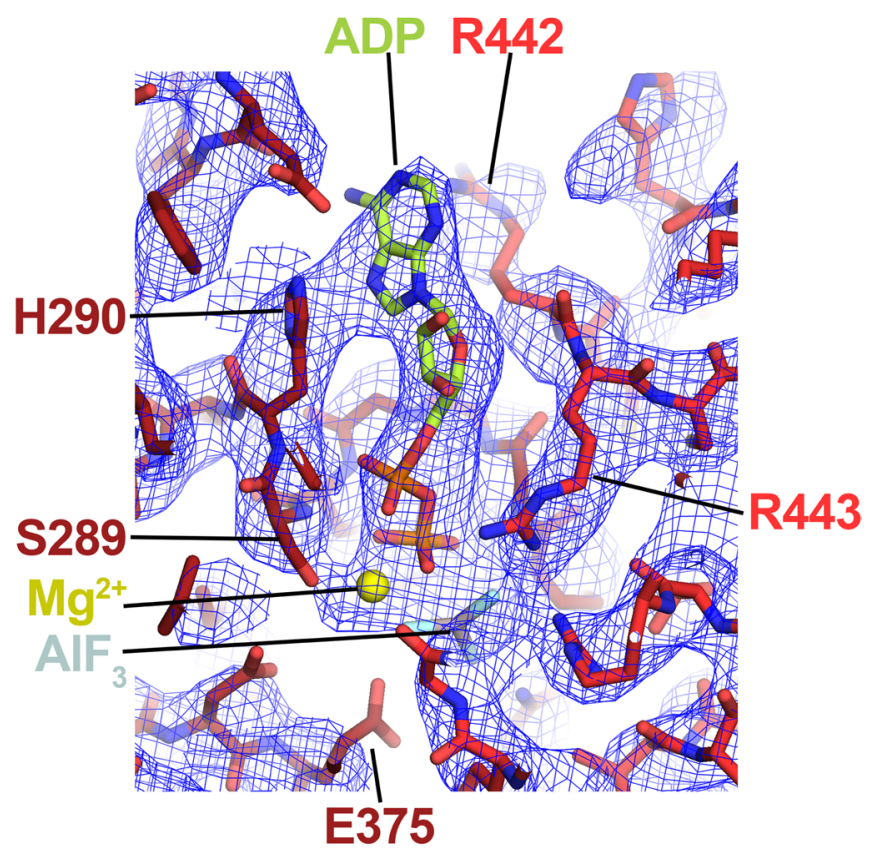

RNA base pairs

B
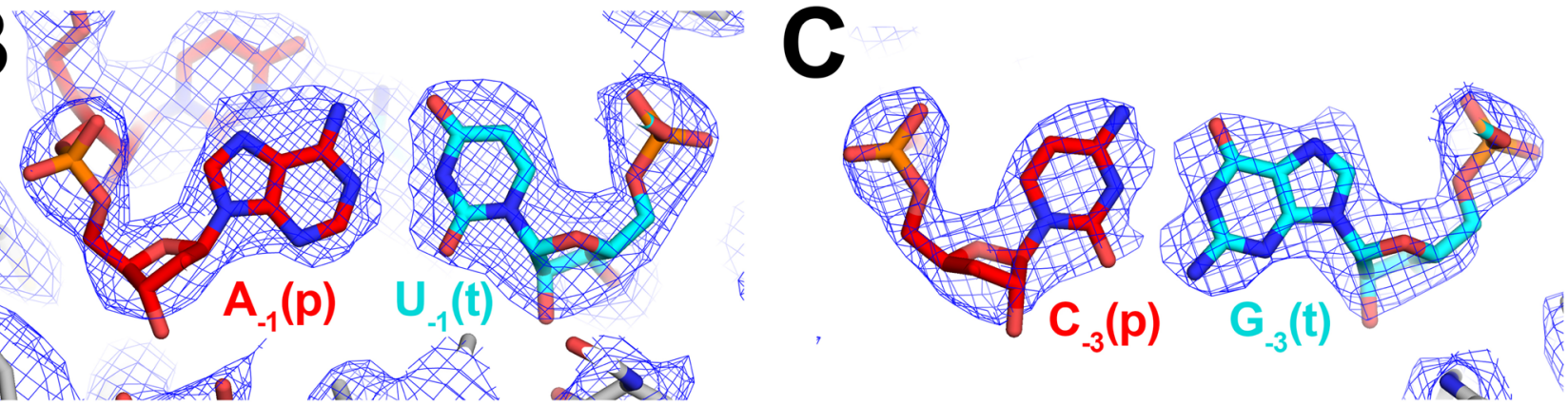

Extended Data Fig. $\mathbf{5}$ | Selected examples of nsp13 - -RTC composite cryo-EM map (map9). a. NiRAN-ADP-Mg2+ bound in the RdRp NiRAN domain. b. AU RNA base pair. c. CG RNA base pair. d. ADP-Mg ${ }^{2+}-\mathrm{AlF}_{3}$ bound to nsp13 

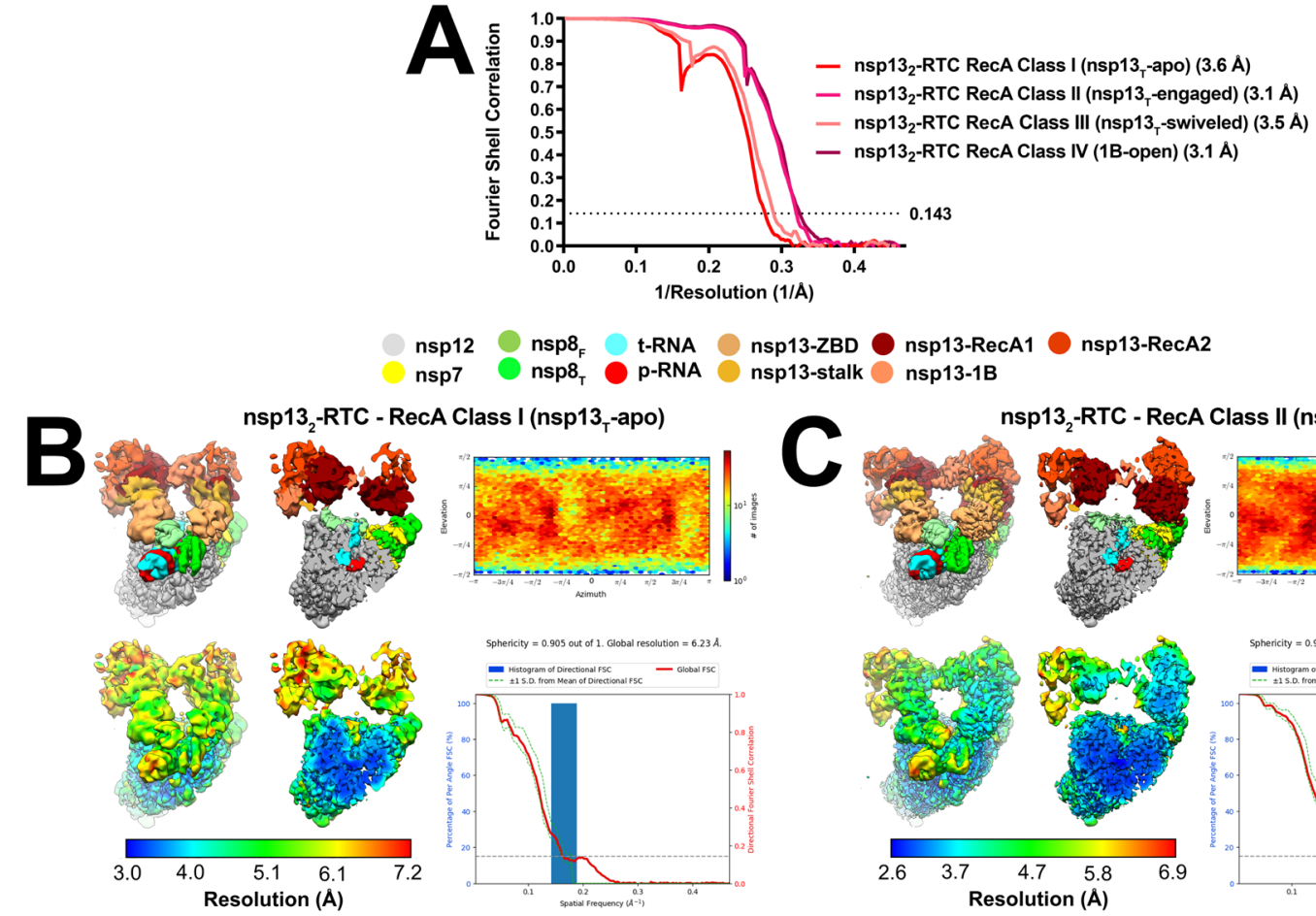

sp13 - -RTC - RecA Class II (nsp13 ${ }_{T}$-engaged)
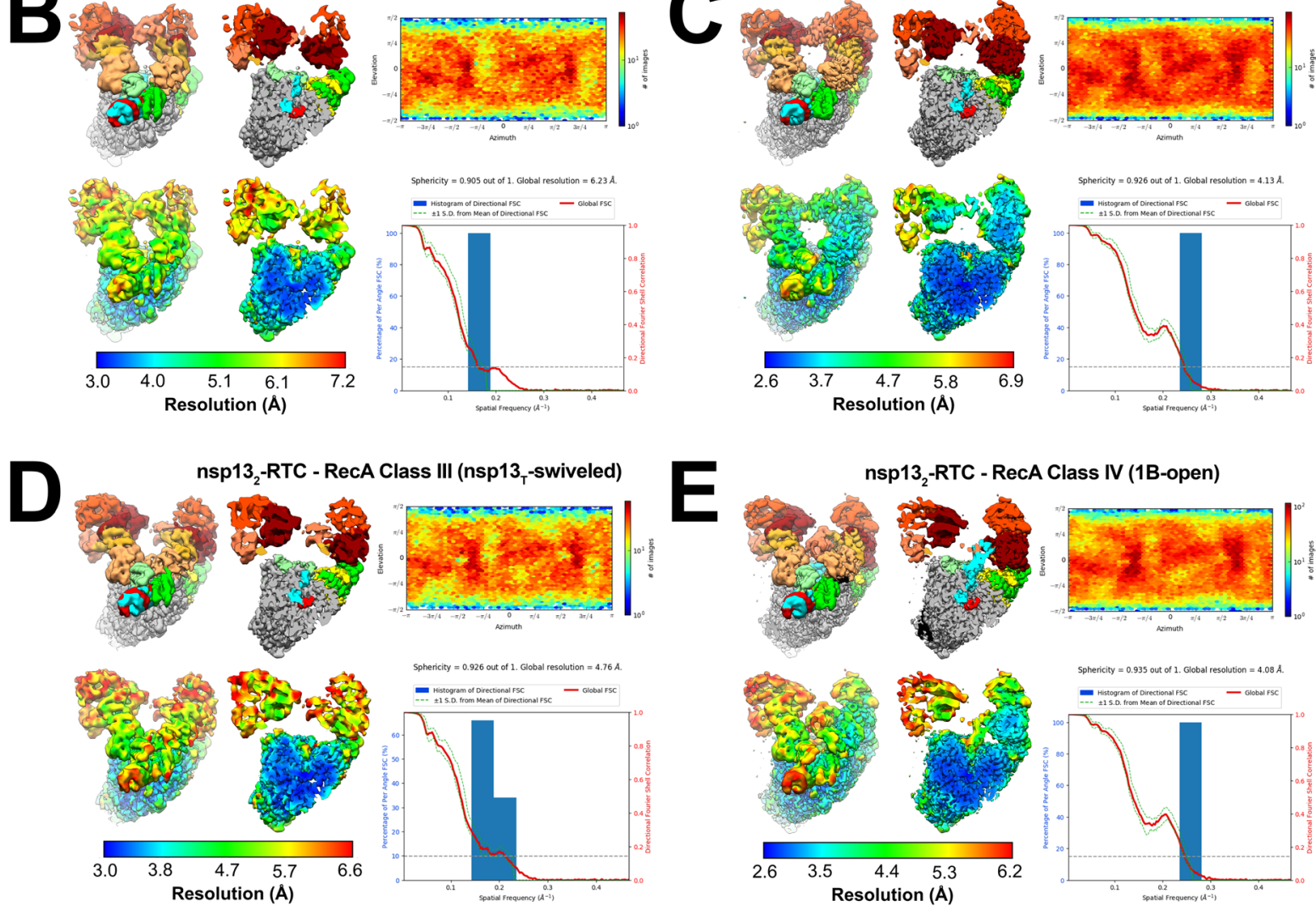

D

Cist
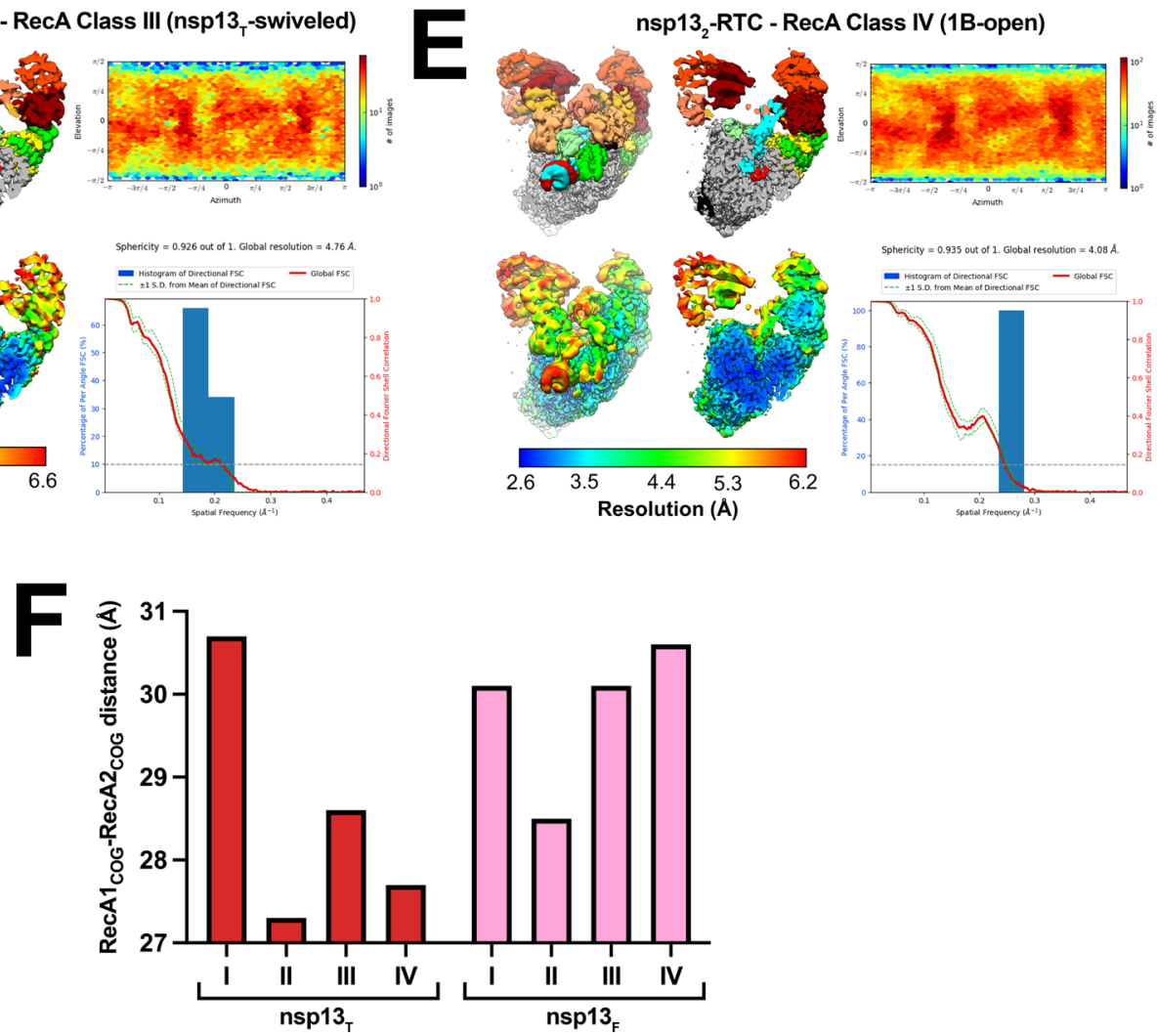

Extended Data Fig. 6 | Cryo-EM of nsp13 2 -RTC classes. a. Gold-standard Fourier-shell correlation (FSC) plot for the four nsp13,-RTC classes calculated by comparing the independently determined half-maps from cryoSPARC ${ }^{5}$. The dotted line represents the 0.143 FSC cutoff. $\mathbf{b}$ - e. (left) The cryo-EM maps filtered by local resolution ${ }^{3}$ are shown. The view on the right is a cross-section. In the top row, the maps are colored by subunit according to the key above. In the bottom row, the maps are colored according to the local resolution (color-key indicated in the bar at the bottom). (top-right) Heat map showing particle orientation distribution, calculated using cryoSPARC ${ }^{5}$. (bottom-right) Directional 3D Fourier shell correlation calculated by 3DFSC (top) ${ }^{4}$. b. nsp13 - -apo (map11). c. nsp13 ${ }_{T}$-engaged (map12). d. Nsp13 ${ }_{T}$-swiveled (map10). e. 1B-open (map13). f. Histogram denoting the distance separating the RecA1 center-of-gravity $\left(\operatorname{Rec} A 1_{c o g}\right.$ ) and the RecA $2_{\operatorname{cog}}$ for $n s p 13_{T}$ (red bars, left) and nsp13 (pink bars, right) for the four nsp13 - RTC classes. 


\section{A}

Open $1 \mathrm{~B}$ domain to closed 1B domain transition; nsp13 in 1B-open state

B

Open $1 \mathrm{~B}$ domain to closed 1B domain transition; nsp13 in nsp13,-swiveled state
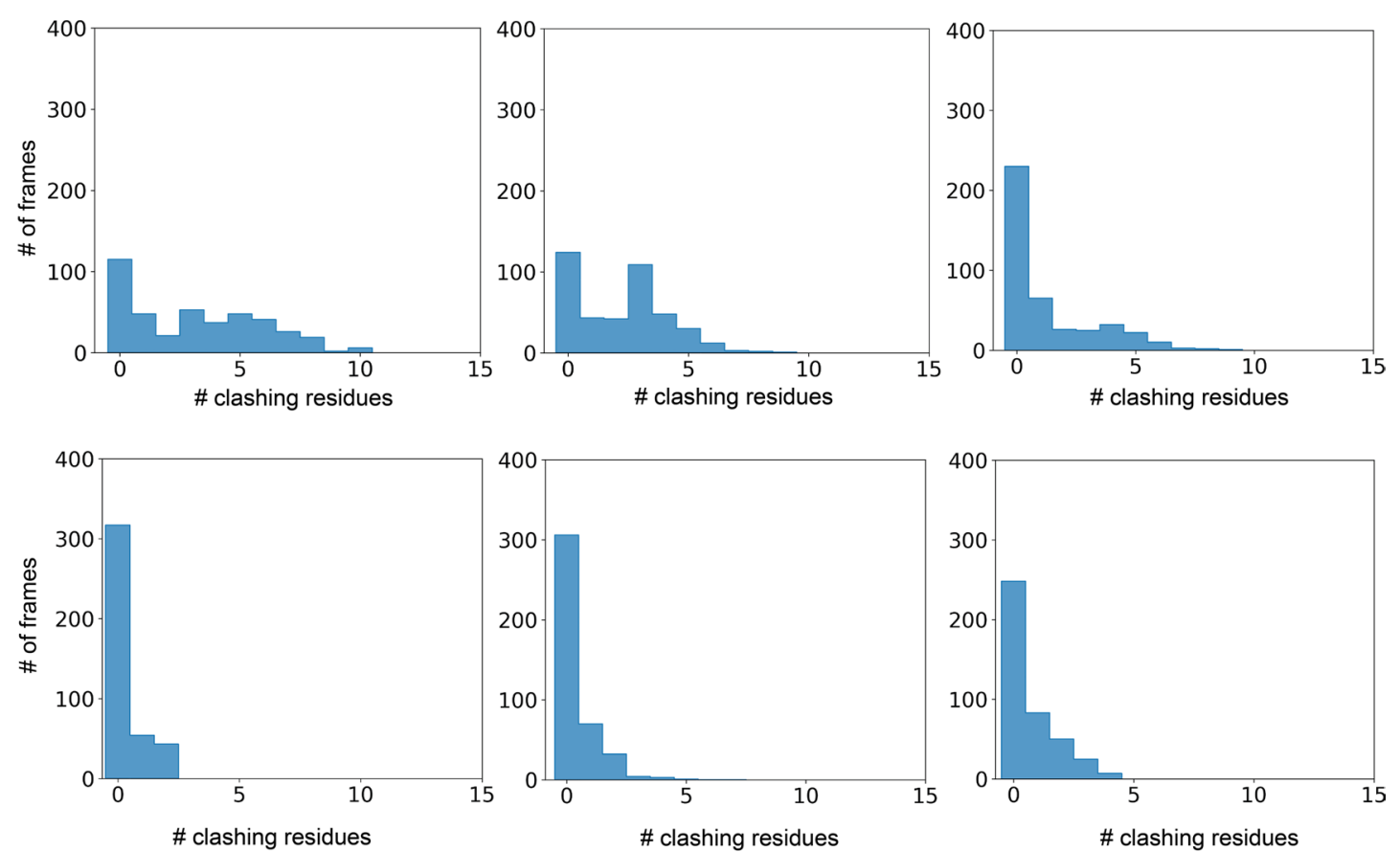

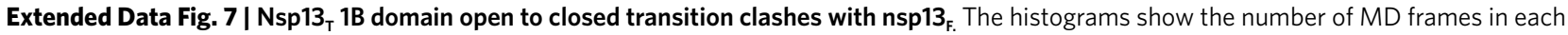
simulation in which nsp13 $3_{T}$ clashes with $n s p 13_{F}$ during the $n s p 13_{T}-1 B$ domain open to closed states. $\mathbf{a} . \mathrm{Nsp} 13_{T}$ in the nsp13 $3_{T}$-engaged position. $\mathbf{b}$. Nsp13 $3_{T}$ in the $n s p 13_{T}$-swiveled position. 

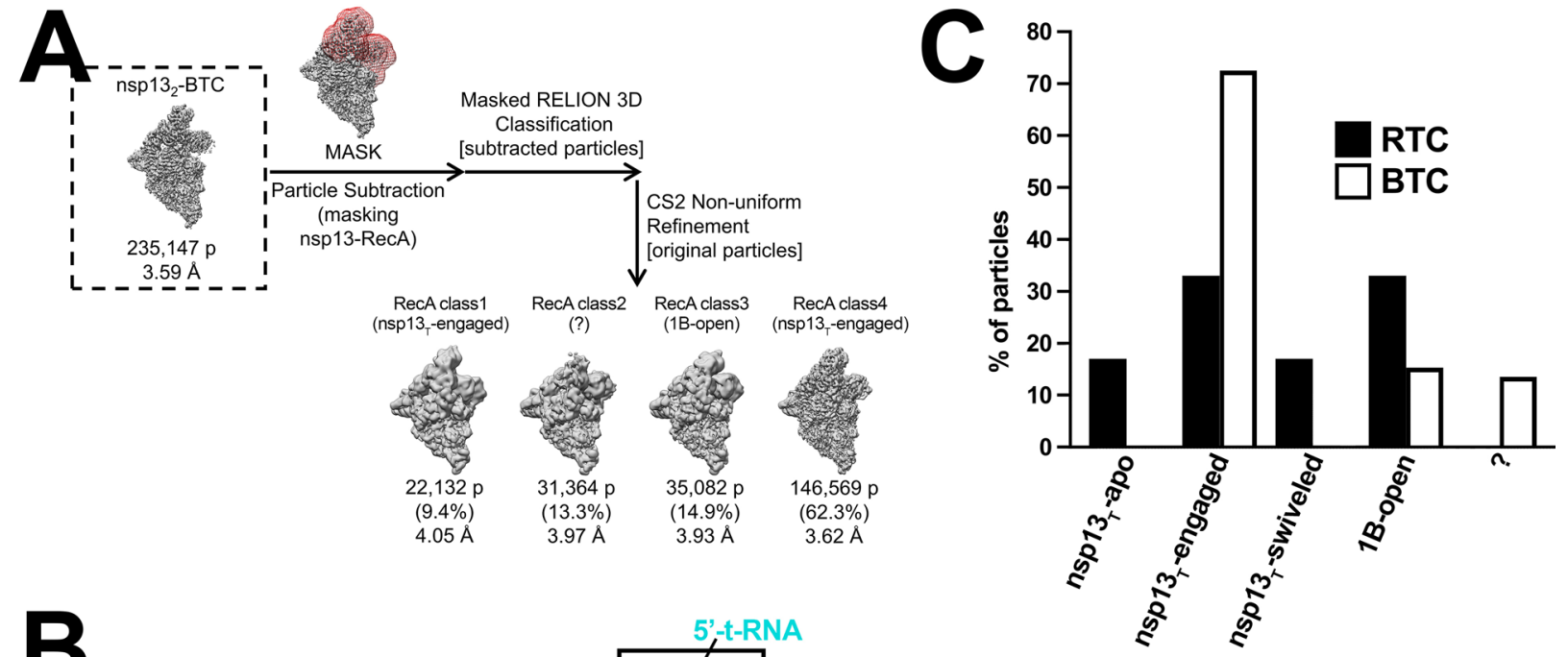

B

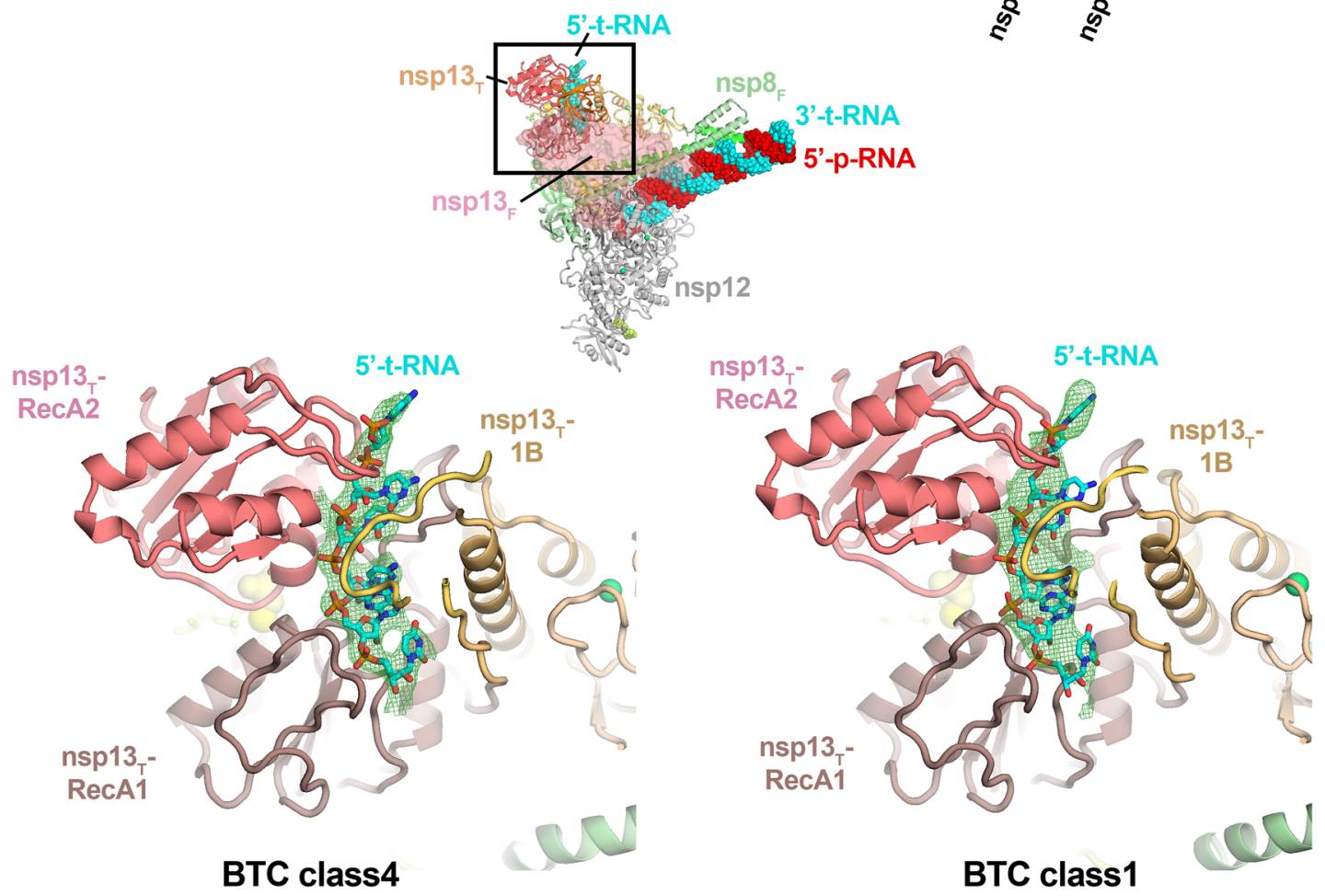

Extended Data Fig. 8 | Classification pipeline for the nsp13 ${ }_{2}-$ BTC dataset and comparison of nsp13 - RTC and nsp13 - -BTC class distribution. a. Focused classification pipeline for $n s p 13_{2}-$ BTC dataset $^{6}$. b. (top-middle) View of the nsp13 - -BTC-class4. Proteins are shown as backbone ribbons except nsp13 is $_{\mathrm{F}}$ shown as a transparent surface. The RNA is shown as spheres. The boxed region is magnified in the views below. (bottom) Magnified views of the nsp13 ${ }_{T}$ RNA binding channel for nsp13 - -BTC-class4 (left) and nsp13 - -BTC-class1 (right). The downstream single-stranded t-RNA is shown as sticks. Cryo-EM difference densities for the downstream single-stranded t-RNA are shown (green mesh). c. Histogram illustrating the nsp13 $3_{2}$-RTC particle distribution for the $n s p 13_{2}-$ RTC (black bars) and nsp13 - BTC (white bars). 


\section{Reporting Summary}

Nature Portfolio wishes to improve the reproducibility of the work that we publish. This form provides structure for consistency and transparency in reporting. For further information on Nature Portfolio policies, see our Editorial Policies and the Editorial Policy Checklist.

\section{Statistics}

For all statistical analyses, confirm that the following items are present in the figure legend, table legend, main text, or Methods section.

$\mathrm{n} / \mathrm{a}$ Confirmed

$\bigotimes$ The exact sample size $(n)$ for each experimental group/condition, given as a discrete number and unit of measurement

$\square$ A statement on whether measurements were taken from distinct samples or whether the same sample was measured repeatedly

$\triangle \square$ The statistical test(s) used AND whether they are one- or two-sided

$\triangle$ Only common tests should be described solely by name; describe more complex techniques in the Methods section.

$\bigotimes \square$ A description of all covariates tested

\ $\square$ A description of any assumptions or corrections, such as tests of normality and adjustment for multiple comparisons A full description of the statistical parameters including central tendency (e.g. means) or other basic estimates (e.g. regression coefficient) AND variation (e.g. standard deviation) or associated estimates of uncertainty (e.g. confidence intervals)

Х For null hypothesis testing, the test statistic (e.g. $F, t, r)$ with confidence intervals, effect sizes, degrees of freedom and $P$ value noted

$\square$ Give $P$ values as exact values whenever suitable.

Х $\square$ For Bayesian analysis, information on the choice of priors and Markov chain Monte Carlo settings

$\bigotimes \square$ For hierarchical and complex designs, identification of the appropriate level for tests and full reporting of outcomes

$\bigotimes \square$ Estimates of effect sizes (e.g. Cohen's $d$, Pearson's $r$ ), indicating how they were calculated

Our web collection on statistics for biologists contains articles on many of the points above.

\section{Software and code}

Policy information about availability of computer code

Data collection

Leginon 3.5

Data analysis Bsoft version 2 (https:///sbr.niams.nih.gov/bsoft/)

Coot 0.8.9.3

cryoSPARC V2

Molprobity 4.5 .1 (http://molprobity.biochem.duke.edu/)

MotionCor2 (https://emcore.ucsf.edu/ucsf-software)

PDBePISA 1.48 (https://www.ebi.ac.uk/pdbe/pisa/) Phenix 1.19 .2

Relion 3.1

PyMOL 2.5.1

ChimeraX 1.2.5

For manuscripts utilizing custom algorithms or software that are central to the research but not yet described in published literature, software must be made available to editors and

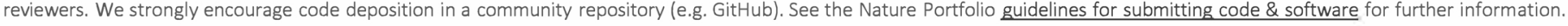


Policy information about availability of data

All manuscripts must include a data availability statement. This statement should provide the following information, where applicable:

- Accession codes, unique identifiers, or web links for publicly available datasets

- A description of any restrictions on data availability

- For clinical datasets or third party data, please ensure that the statement adheres to our policy

Data and code availability. All unique/stable reagents generated in this study are available without restriction from one of the Lead Contacts, Seth A. Darst (darst@rockefeller.edu).The cryo-EM density maps and atomic coordinates have been deposited in the EMDataBank and Protein Data Bank as follows: nsp131-RTC (EMD-24431, 7RE2), nsp132-RTC (composite) (EMD-24430, 7RE1), (nsp132-RTC)2 (EMD-24432, 7RE3), nsp132-RTC (nsp13.1-apo) (EMD-24428, 7RDZ), nsp132-RTC (nsp13.1-engaged) (EMD-24427, 7RDY), nsp132-RTC (nsp13.1-swiveled) (EMD-24429, 7REO), nsp132-RTC (1B-open) (EMD-24426, 7RDX). The MD trajectories described in this work are available at https://www.deshawresearch.com/downloads/download_trajectory_sarscov2.cgi/.

\section{Field-specific reporting}

Please select the one below that is the best fit for your research. If you are not sure, read the appropriate sections before making your selection.

$\bigotimes$ Life sciences

Behavioural \& social sciences

Ecological, evolutionary \& environmental sciences

For a reference copy of the document with all sections, see nature.com/documents/nr-reporting-summary-flat.pdf

\section{Life sciences study design}

All studies must disclose on these points even when the disclosure is negative.

$\begin{array}{ll}\text { Sample size } & \begin{array}{l}\text { The cryo-EM data collection resulted in 661,105 particle images used for subsequent processing (see Fig. S2). This was deemed sufficient } \\ \text { based on the quality of the cryo-EM maps. }\end{array} \\ \text { Data exclusions } & \begin{array}{l}\text { Particle images were excluded according to critera encoded in cryoSPARC and Relion. } \\ \text { Replication }\end{array} \\ \text { Randomization } & \text { The structural analysis was not replicated. } \\ \text { Blinding structural analysis was not randomized }\end{array}$

\section{Reporting for specific materials, systems and methods}

We require information from authors about some types of materials, experimental systems and methods used in many studies. Here, indicate whether each material, system or method listed is relevant to your study. If you are not sure if a list item applies to your research, read the appropriate section before selecting a response.

\begin{tabular}{l|l} 
Materials \& experimental syster \\
\hline n/a Involved in the study \\
$\square$
\end{tabular}

\begin{tabular}{|c|c|}
\hline \multicolumn{2}{|c|}{ Methods } \\
\hline$n / a$ & Involved in the study \\
\hline$\bigotimes$ & ChIP-seq \\
\hline$\bigotimes$ & Flow cytometry \\
\hline$\bigotimes$ & MRI-based neuroimaging \\
\hline
\end{tabular}

